\title{
Propriétés mécaniques de l'argile des Flandres à Dunkerque et à Calais
}

H. JOSSEAUME

LCPC, 58, bd Lefebvre, 75732 Paris Cedex 15
L'article rend compte de l'étude des propriétés de l'argile des Flandres rencontrée à Dunkerque et à Calais sous une couche de sable d'une trentaine de mètres

d'épaisseur. Les caractéristiques mécaniques de cette argile raide, très plastique et fortement surconsolidée ont été déterminées à partir d'essais de laboratoire et en place effectués dans le cadre de la reconnaissance des deux sites où des murs de quai de grande hauteur devaient être construits, ainsi que dans le cadre de recherches sur le comportement des argiles raides. Ces essais, qui couvrent presque toute la gamme des essais de sol couramment réalisés, ont permis d'obtenir les paramètres de résistance au cisaillement de l'argile, certains paramètres de déformabilité ainsi que le coefficient de pression du sol au repos. Des relations empiriques exprimant la cohésion non drainée et les modules de déformation en fonction de la pression de consolidation ont en outre été établies. D'autre part, la fiabilité de la détermination de la cohésion non drainée à partir d'essais triaxiaux UU et de certains essais en place (essais au pressiomètre Ménard, essais de pénétration statique et essais au dilatomètre Marchetti) a également été discutée.

\section{Engineering properties of the Flanders clay at Dunkirk and Calais}

The paper deals with the properties of the Flanders clay lying at Dunkirk and Calais under a sand layer 30 meter thick or so. The engineering properties of this stiff, highly plastic and heavily overconsolidated clay have been derived from laboratory and in situ soil tests carried out as a part of the investigation of the two sites where the construction of high quay walls was planned and for research purposes about the behaviour of stiff clays. From these tests it was possible to derive the shear strength parameters of the clay, some deformability parameters and the coefficient of earth pressure at rest. The interpretation of the tests also led to empirical relationships linking undrained shear strength and deformation moduli to consolidation pressure. Besides, the reliability of undrained shear strength values derived from triaxial UU tests and from in situ tests such as Ménard pressuremeter, static penetration and Marchetti dilatometer tests has also been discussed.
} 


\section{Introduction}

L'argile des Flandres est la principale formation géologique de la plaine des Flandres, dont elle constitue le soubassement. Elle forme une couche de forte épaisseur, généralement affleurante, sauf dans la plaine côtière, large de $10 \mathrm{~km}$ à $20 \mathrm{~km}$, où elle est recouverte par des formations sableuses dont l'épaisseur peut atteindre une trentaine de mètres. Dans cette zone, l'argile des Flandres se présente sous la forme d'un matériau raide et gonflant dont les caractéristiques géotechniques sont relativement constantes.

L'étude des propriétés de cette formation a été entreprise dans le cadre des recherches des Laboratoires des ponts et chaussées sur le comportement des sols raides. Elle avait pour objectif la détermination des paramètres de résistance au cisaillement et de déformabilité qui caractérisent le comportement de l'argile ainsi que l'étude de l'évolution de ses caractéristiques non drainées due au gonflement. Cette évolution pouvant affecter la stabilité de certains murs de quai dragués, fichés dans l'argile (murs soumis à d'importantes sollicitations accidentelles survenant longtemps après la mise en service des ouvrages, murs en cours de rempiétement), son étude a fait l'objet de contrats de recherche entre le STCPMVN et le Port autonome de Dunkerque, d'une part, et le LCPC, d'autre part.

L'étude de l'argile des Flandres, dont il est rendu compte ci-après, s'appuie sur une importante campagne d'essais de laboratoire, réalisée au LCPC en 1986 et 1987, dans le cadre des contrats de recherche mentionnés précédemment et dans le cadre d'une thèse (Hieng, 1991), sur des échantillons prélevés dans la zone portuaire de Dunkerque. Elle intègre également les résultats de nombreux essais de laboratoire et d'essais en place effectués, principalement par le Laboratoire régional des ponts et chaussées de Lille, lors des campagnes de reconnaissance qui ont précédé la construction de deux importants ouvrages portuaires, le quai à pondéreux ouest (QPO) construit à Dunkerque en 1982 (Port autonome de Dunkerque, 1981) et le quai en eau profonde (QEP) construit à Calais en 1989 (Neveu et al., 1994).

L'analyse des essais œdométriques effectués au LCPC sur l’argile prélevée à Dunkerque, qui était principalement orientée vers la détermination de la pression de préconsolidation du sol en place, a déjà fait l'objet d'une publication détaillée (Josseaume et al., 1991). Aussi le présent article est-il essentiellement consacré à l'étude des caractéristiques de résistance au cisaillement et de déformabilité de l'argile des Flandres et des effets du gonflement.

\section{L'argile des Flandres dans la plaine côtière : géologie et identification}

Les campagnes de reconnaissance effectuées à Dunkerque entre 1975 et 1985 dans le cadre de l'étude du QPO ont établi que, dans la zone intéressée par cet ouvrage long de $450 \mathrm{~m}$, le toit de l'argile des Flandres se trouve à une profondeur moyenne de $32 \mathrm{~m}$ par rap- port au terrain naturel. Des sondages profonds ont par ailleurs montré que l'épaisseur de la couche d'argile est de l'ordre de $90 \mathrm{~m}$. Le recouvrement de l'argile est constitué par les alluvions du Flandrien, formées de sable fin plus ou moins limoneux traversé localement de passées limoneuses. Ces alluvions sont baignées par une nappe dont la surface se situe à environ $5 \mathrm{~m}$ de profondeur par rapport au niveau moyen du terrain naturel. Les sondages, exécutés à quelques centaines de mètres du QPO afin de prélever les échantillons nécessaires à l'étude en laboratoire réalisée au LCPC en 1986 et 1987 confirment les résultats précédents. A l'emplacement de ces sondages, qui ont atteint une profondeur de $45 \mathrm{~m}$, l'argile a été rencontrée à $32 \mathrm{~m}$ de profondeur et la nappe du sable flandrien à $5 \mathrm{~m}$. En outre, la reconnaissance du site du QEP à Calais a mis en évidence une coupe géologique très semblable : la hauteur de recouvrement de l'argile par le sable flandrien est de l'ordre de $30 \mathrm{~m}$ et la nappe de cette formation se situe à une profondeur de $5 \mathrm{~m}$.

L'examen des échantillons prélevés lors des différentes campagnes de reconnaissance montre que l'argile se présente sous la forme d'un matériau gris foncé d'apparence homogène mais qui est traversé de microfissures qui le découpent en polyèdres irrégulièrement distribués. Les valeurs des paramètres d'état et d'identification, mesurées sur ces échantillons, sont présentées dans le tableau I. La quasi-identité des valeurs obtenues à Dunkerque et à Calais met en évidence la similitude des propriétés de l'argile sur ces deux sites, ce qui légitime la prise en compte dans l'étude, de l'ensemble des résultats relatifs à ceux-ci. Les caractéristiques d'état et d'identification indiquent que l'argile des Flandres est une argile très consistante et très plastique représentée par les symboles $\mathrm{A}$, et $\mathrm{L}$. dans la classification des LPC. Par ailleurs, les essais de gonflement et les essais œedométriques à haute pression réalisés sur ce matériau (Josseaume et al., 1991)

TABLEAU1 Plages de variation et valeurs moyennes des paramètres d'état et d'identification. Ranges and average values of the state and identification parameters.

\begin{tabular}{|c|c|c|c|}
\hline Site & DUNK & RQUE & CAIAIS \\
\hline Origine des données & LPPC de Lille & LCPC & LRPC de Lille \\
\hline Étude & Quaiłá pondéreux & $\begin{array}{l}\text { Comportement } \\
\text { de l'argile }\end{array}$ & $\begin{array}{l}\text { Quaien } \\
\text { eau profonde }\end{array}$ \\
\hline $\begin{array}{l}\text { Limité de liquicitié } \\
\left.\qquad w_{L}(9)\right)\end{array}$ & $\begin{array}{c}62 \cdot 96 \\
75\end{array}$ & $\frac{63-75}{71}$ & $\begin{array}{l}69-83 \\
75,4\end{array}$ \\
\hline $\begin{array}{c}\text { Indice de } \\
\text { plasticitél, }{ }_{i}(\%)\end{array}$ & $\begin{array}{c}32-57 \\
41\end{array}$ & $\begin{array}{c}40-47 \\
44\end{array}$ & $\begin{array}{c}3446 \\
40\end{array}$ \\
\hline $\begin{array}{l}\text { Teneur en eau } \\
\qquad \mathrm{w}(\%)\end{array}$ & $\begin{array}{l}26-35 \\
29,6\end{array}$ & $\begin{array}{l}26-31 \\
28,6\end{array}$ & $\begin{array}{l}2434 \\
28,9\end{array}$ \\
\hline $\begin{array}{l}\text { Poids volumique } \\
\gamma\left(\mathrm{kN} / \mathrm{m}^{2}\right)\end{array}$ & $\begin{array}{c}19,6 \cdot 20,3 \\
19,8\end{array}$ & $\begin{array}{c}19,3-21,2 \\
19,9\end{array}$ & $\begin{array}{c}19,4-20,4 \\
19,9\end{array}$ \\
\hline $\begin{array}{l}\text { Poids volumique sec } \\
\qquad \gamma_{4}\left(\mathrm{kN} / \mathrm{m}^{3}\right)\end{array}$ & $\begin{array}{c}13,8-16,1 \\
15,3\end{array}$ & $\begin{array}{c}14,9-16,3 \\
15,5\end{array}$ & $\begin{array}{c}14,5 \cdot 16,3 \\
15,4\end{array}$ \\
\hline $\begin{array}{l}\text { Poids volumique des } \\
\text { particules ys }\left(\mathrm{kN} / \mathrm{m}^{2}\right)\end{array}$ & & $\begin{array}{c}27-28,5 \\
27,5\end{array}$ & \\
\hline Teneur en argile $(\%)$ & & 56 & \\
\hline
\end{tabular}


mettent en évidence son caractère gonflant (le gonflement relatif mesuré au cours d'essais de gonflement libre est d'environ $8 \%$ ) et sa forte surconsolidation, la pression de préconsolidation au niveau du toit de la couche étant supérieure à $3000 \mathrm{kPa}$ ( $\mathrm{cf}$. \$ 3.1.2). Le caractère gonflant de l'argile est lié à la forte teneur en montmorillonite de sa fraction argileuse, son état de surconsolidation étant une conséquence de son histoire géologique, qui est résumée ci-après.

L'argile des Flandres s'est déposée à l'Yprésien (début de l'Éocène de l'ère tertiaire) dans un golfe marin qui s'étendait sur toute la zone où se situent actuellement le Nord de la France, la Belgique et le Sud-Est de l'Angleterre. Elle a ensuite été recouverte par des formations tertiaires dont la sédimentation s'est poursuivie jusqu'au Pliocène supérieur. La surface du sol se situait alors probablement à environ $200 \mathrm{~m}$ audessus de la surface actuelle de l'argile, cette hauteur de recouvrement étant estimée par référence à l'altitude de la butte-témoin du Mont Cassel. Les formations recouvrant l'argile ainsi que la partie supérieure de celle-ci ont ensuite été érodées. Ce processus d'érosion a été suivi au Quaternaire, par le dépôt des alluvions du Flandrien qui exercent actuellement sur l'argile restant en place une contrainte verticale effective très inférieure à celle qui lui a été appliquée au Pliocène supérieur.

\section{3}

\section{Étude expérimentale des propriétés mécaniques de l'argile des Flandres}

L'étude des propriétés mécaniques de l'argile des Flandres tient compte, d'une part, des résultats des recherches en laboratoire effectuées au LCPC et, d'autre part, des résultats d'essais courants de laboratoire et d'essais en place, réalisés à Dunkerque et à Calais lors des campagnes de reconnaissance du QPO et du QEP auxquelles le LRPC de Lille a pris une part prépondérante.

\section{1}

\section{Étude en laboratoire du comportement de l'argile}

\section{4 \\ Généralités}

L'étude en laboratoire a comporté la réalisation d'essais cedométriques, d'essais triaxiaux de divers types et d'essais au pénétromètre de laboratoire. Ces essais ont été effectués sur des éprouvettes découpées dans des échantillons $\alpha$ intacts $)$ extraits de trois sondages voisins implantés à quelques centaines de mètres du QPO. Ces échantillons de diamètre $96,7 \mathrm{~mm}$ ont été prélevés entre $34 \mathrm{~m}$ et $45 \mathrm{~m}$ de profondeur (c'est-à-dire de $2 \mathrm{~m}$ à $13 \mathrm{~m}$ au-dessous du toit de l'argile) au moyen d'un carottier à triple enveloppe (carottier à câble SKL 146, adaptation Mazier). Ils ont été conditionnés en vue de leur conservation et ont été stockés en chambre humide à une température de $8{ }^{\circ} \mathrm{C}$ avant exécution des essais.

\subsection{0}

\section{Essais œedométriques}

La compressibilité de l'argile a été étudiée à partir de dix-sept essais cedométriques effectués au moyen de l'appareillage courant, qui ne permet pas d'appliquer à l'éprouvette des contraintes supérieures à environ $3000 \mathrm{kPa}$, et de trois essais œdométriques à haute pression dans lesquels le sol est soumis à une contrainte maximale de $22000 \mathrm{kPa}$. L'analyse des résultats ayant montré que des valeurs représentatives des paramètres de compressibilité d'une argile raide ne peuvent être obtenues qu'à partir des essais à haute pression (Josseaume et al,, 1991), seuls les résultats de ces derniers ont été retenus (tableau II). Ils font apparaître que la pression de préconsolidation $\sigma_{\text {p }}^{\prime}$ est d'environ $3200 \mathrm{kPa}$ (moyenne des valeurs obtenues) à $11 \mathrm{~m}$ au-dessous du toit de la couche, c'est-à-dire de l'ordre de $3100 \mathrm{kPa}$ au niveau de celui-ci.

TABLEAU II Essais cedométriques à haute pression. Paramètres d'état des éprouvettes et principaux résultats d'essais. High pressure oedometer tests, State parameters of the specimens and main test results.

\begin{tabular}{c|c|c|c|c|c}
\hline $\begin{array}{c}\text { Protondeur } \\
(\mathrm{m})\end{array}$ & $\begin{array}{c}\gamma_{i} \\
\left(\mathrm{kN} / \mathrm{m}^{3}\right)\end{array}$ & $\begin{array}{c}w \\
\%\end{array}$ & $\begin{array}{c}\sigma^{\prime} \\
(\mathrm{kPa})\end{array}$ & $\mathrm{C}_{\mathrm{c}}$ & $\mathrm{C}_{\mathrm{s}}$ \\
\hline 42,90 & 15,4 & 30,5 & 3400 & 0,34 & 0,12 \\
\hline 42,95 & 16 & 26,2 & 3400 & 0,29 & 0,10 \\
\hline 43 & 15,8 & 27,6 & 2800 & 0,31 & 0,10 \\
\hline
\end{tabular}

\section{3in:3}

Essais triaxiaux

\section{3inain}

Programme d'essais

L'objectif des essais était de déterminer les valeurs des paramètres de résistance au cisaillement et de déformabilitê qui régissent les comportements à court terme et à long terme de l'argile sollicitée par un écran de soutènement fiché dans celle-ci. Dans cette optique, on a donc réalisé des essais drainés et non drainés dans lesquels les éprouvettes ont été cisaillées à pression latérale constante soit en compression, soit en extension, ces modes de déformation s'apparentant respectivement aux modes de déformation de l'argile en place à l'amont et à l'aval d'un écran de soutènement. En effet, lors du dragage qui provoque un déplacement de l'écran vers l'aval, si l'on néglige les distorsions introduites par la rotation des contraintes, les mécanismes de déformation du sol en place peuvent être schématisés comme suit : le sol situé à l'amont est déchargé latéralement à contrainte verticale constante et subit de ce fait une compression verticale, tandis qu'à l'aval le sol, qui est déchargé verticalement et comprimé latéralement, subit une extension verticale.

Le programme d'essais, qui intéressait un total de 46 éprouvettes, a comporté :

-2 essais UU (essais non drainés en compression sur des éprouvettes non reconsolidées en laboratoire); 
- 11 essais CIU (essais non drainés en compression sur des éprouvettes consolidées sous contrainte isotrope) ;

- 12 essais EIU (essais non drainés en extension sur des éprouvettes consolidées sous contrainte isotrope) ;

- 15 essais CID (essais drainés en compression sur des éprouvettes consolidées sous contrainte isotrope) ;

- 6 essais EID (essais drainés en extension sur des éprouvettes consolidées sous contrainte isotrope).

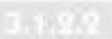

\section{Procédures d'essai}

Les essais ont été effectués sur des éprouvettes de $38,2 \mathrm{~mm}$ de diamètre et de $76,4 \mathrm{~mm}$ de hauteur, découpées manuellement sur un touret vertical.

Chaque éprouvette a été montée à sec, c'est-à-dire entre deux pierres poreuses sèches, dans une cellule triaxiale équipée de deux sorties de drainage connectées à la pierre poreuse supérieure, cette disposition permettant une saturation complète et rapide du circuit de drainage. Au cours du montage, un drain ajouré en papier filtre était interposé entre la surface latérale de l'éprouvette et la membrane. Dans le cas d'un essai en extension, une cellule munie d'un piston dont la partie inférieure peut être solidarisée avec le chapeau de l'éprouvette par une liaison à rotule était utilisée.

Chaque essai a commencé par la mesure de la contrainte isotrope de gonflement $\sigma_{\text {git }}^{\prime}$ qui, dans le cas d'une argile saturée, est égale, au signe près, à la pression interstitielle initiale négative $u_{\text {qui }}$ règne dans l'éprouvette non chargée et par suite à la contrainte isotrope effective appliquée à cette dernière. La mesure de $\sigma_{p i}^{\prime}$ a été réalisée comme indiqué ci-dessous :

- application à l'éprouvette d'une contrainte isotrope de l'ordre de la contrainte verticale effective du sol en place $\sigma^{\prime}$ au niveau de prélèvement, afin d'éviter tout gonflement pendant la saturation du circuit de drainage;

- saturation du circuit de drainage et fermeture des sorties de drainage à l'exception de l'une des sorties connectées à la pierre poreuse inférieure ;

- ajustement progressif de la pression dans la cellule à une valeur telle que l'éprouvette ne subisse aucune variation de volume, la nullité des variations de volume étant contrôlée au moyen d'un indicateur de zéro monté sur la sortie de drainage laissée en communication avec l'atmosphère (Berre, 1981). A l'équilibre, la pression dans la cellule est égale à $\sigma_{\text {pip }}$.

Tous les essais ont été effectués sous une contrepression de $400 \mathrm{kPa}$. Celle-ci a été appliquée en quatre paliers $(50 \mathrm{kPa}, 100 \mathrm{kPa}, 200 \mathrm{kPa}$ et $400 \mathrm{kPa})$, soit immédiatement après la mesure de $\sigma^{\prime}$ dans le cas des essais UU et d'une partie des essais de type consolidé, soit après la phase de consolidation dans le cas des autres essais.

La procédure consistant à consolider les éprouvettes préalablement à l'application de la contre-pression (le circuit de drainage était alors à la pression atmosphérique) a été mise en cuvre au début de l'étude. Il est apparu qu'elle avait pour inconvénient d'augmenter la durée de la consolidation (efficacité faible ou nulle du drain latéral) et, dans certains cas, de conduire à des courbes de consolidation difficilement interprétables (l'expulsion d'eau par l'éprouvette au cours des quatre à dix premières heures étant suivie d'une absorption beaucoup plus lente), aussi a-t-elle été abandonnée au bénéfice de celle qui consiste à réaliser la consolidation sous contre-pression.

Les éprouvettes sur lesquelles ont été effectuées les essais de type CIU, CID, EIU et EID ont été consolidées sous une contrainte isotrope effective $\sigma^{\prime}$ comprise entre $50 \mathrm{kPa}$ et $790 \mathrm{kPa}$. Dans la première partie de l'étude, les éprouvettes ont été consolidées en une seule phase. Par la suite, pour compenser, dans une certaine mesure, les effets de la " déconsolidation ») de l'argile dans le temps (mise en évidence par la diminution des valeurs de $\sigma^{\prime}$ mesurées au fur et à mesure du déroulement de l'étude), la consolidation sous $\sigma^{\prime}$ a été systématiquement précédée d'une phase de reconsolidation sous une contrainte isotrope effective $\sigma_{c o}^{\prime}$ de $470 \mathrm{kPa}$ sensiblement égale à $\sigma_{\text {vó }}^{\prime}$

Pendant la (ou les) phase(s) de consolidation, on a relevé en fonction du temps, non seulement les variations de volume de l'éprouvette, mais également ses déformations axiales. Pour mesurer ces dernières, le piston de la cellule a été maintenu au contact du chapeau de l'éprouvette en lui appliquant, au moyen d'un plateau chargé par des poids, adapté temporairement à son extrémité supérieure, une force qui équilibrait la poussée ascendante du fluide cellulaire. Dans ces conditions, la déformation axiale était égale au déplacement du piston.

Toutes les éprouvettes ont été cisaillées à contrainte latérale et à vitesse de déformation axiale constantes. La vitesse de déformation axiale adoptée pour toutes les éprouvettes était de $0,047 \% / \mathrm{h}$. Cette valeur, qui correspond à la plus petite vitesse des presses utilisées $(0,6 \mu \mathrm{m} / \mathrm{min})$, a été choisie afin d'obtenir des valeurs élevées du degré de dissipation de la pression interstitielle dans les essais drainés et du degré d'uniformisation de celle-ci dans les essais non drainés. Ces derniers ont tous été réalisés avec mesure de la pression interstitielle au niveau de la pierre poreuse inférieure.

\section{3isk:3}

\section{Résultats des essais}

Les principales grandeurs caractérisant l'état et le comportement des éprouvettes lors des différentes phases des essais sont reportées dans les tableaux III et IV, pour l'ensemble des essais dont les résultats ont été retenus. De plus, des courbes représentant les variations du déviateur $\sigma_{-}-\sigma_{\text {e }}$ en fonction de la déformation axiale $\varepsilon_{\text {a }}$ et les variations de l'accroissement $\Delta u$ de la pression interstitielle ou celles de la déformation volumique $\varepsilon$ pendant la phase de cisaillement sont représentées pour chaque type d'essai sur les figures 1 à 5 . Ces résultats appellent les commentaires formulés ci-après.

L'examen des valeurs de $\sigma_{\text {gij }}^{\prime}$ mesurées au début de chaque essai met en évidence leur diminution avec le temps qui s'écoule entre le prélèvement du sol et l'exécution des essais (Fig. 6). En effet, on constate que $\sigma^{\prime}$ quí est de l'ordre de $90 \%$ de la contrainte verticale effective en place $\sigma_{\text {vo }}^{\prime}$ pour les deux premières mesures (cf. tableau III, essais 1 et 3 ) réalisées environ cinq mois après le prélèvement des échantillons, décroît d'environ $50 \%$ dans les deux mois suivant celles-ci et se stabilise lentement à une valeur de l'ordre de 25 à $30 \%$ de $\sigma_{\text {yo }}^{\prime}$. La contrainte isotrope effective à laquelle sont soumises les éprouvettes non chargées étant sensiblement égale à $\sigma_{\mathrm{gi}}^{\prime}$ (sol saturé), la décroissance de ce paramètre 
TABLEAU III Essais triaxiaux non drainés (UU, CIU, EIU). Paramètres d'état des éprouvettes et principaux résultats d'essais.

Undrained triaxial tests (UU, CIU, EIU). State parameters of the specimens and main test results.

\begin{tabular}{|c|c|c|c|c|c|c|c|c|c|c|c|c|c|c|c|c|c|c|}
\hline \multirow{2}{*}{\multicolumn{2}{|c|}{ Essaí }} & \multicolumn{4}{|c|}{ Éprouvette } & \multirow{2}{*}{\multicolumn{2}{|c|}{$\begin{array}{l}\text { Reconsolidation } \\
\text { sous } \\
\sigma_{\text {cof }}=470 \mathrm{kPa}\end{array}$}} & \multirow{2}{*}{\multicolumn{3}{|c|}{$\begin{array}{c}\text { Consolidation } \\
\text { sous } \sigma_{c}^{\prime}\end{array}$}} & \multicolumn{8}{|c|}{ Cisaillement } \\
\hline & & \multirow{2}{*}{\begin{tabular}{c|} 
Profinder \\
2 \\
(m)
\end{tabular}} & \multicolumn{3}{|c|}{ feterintial } & & & & & & \multicolumn{4}{|c|}{ Nowiende cillonation } & \multicolumn{4}{|c|}{ Peptate } \\
\hline Type & $\mathrm{N}^{\circ}$ & & $\begin{array}{c}\gamma_{k} \\
\left(\mathrm{KNN} / \mathrm{m}^{3}\right)\end{array}$ & $\begin{array}{c}\text { W } \\
(\%)\end{array}$ & $\begin{array}{c}\sigma_{g}^{\prime} \\
(\mathrm{kPa})\end{array}$ & $\begin{array}{l}\varepsilon_{00} \\
(\%)\end{array}$ & $\begin{array}{l}\varepsilon_{\infty} \\
(\%)\end{array}$ & $\begin{array}{c}\sigma_{x}^{\prime} \\
(\mathrm{kPa})\end{array}$ & $\begin{array}{l}\varepsilon_{\mathrm{ve}} \\
(\%)\end{array}$ & $\begin{array}{l}\varepsilon_{m} \\
(\%)\end{array}$ & $\begin{array}{l}E_{\text {uazs }} \\
\text { (MPa) }\end{array}$ & $\begin{array}{c}E_{\text {vos }} \\
\text { (MPa) }\end{array}$ & $\begin{array}{c}E_{w:} \\
(\mathrm{MPa} a)\end{array}$ & $\begin{array}{c}E_{c s 0} \\
(\mathrm{MPa})\end{array}$ & $\begin{array}{l}\varepsilon_{s t} \\
(\%)\end{array}$ & $\begin{array}{c}\left(\sigma_{y}-\sigma_{t}\right)_{f} \\
(\mathrm{kPa})\end{array}$ & $\begin{array}{c}\mathrm{Au} \mathrm{u}_{\mathrm{f}} \\
(\mathrm{kPa})\end{array}$ & $\begin{array}{l}\alpha \\
\text { (9) }\end{array}$ \\
\hline UU & $\begin{array}{l}1 \\
3\end{array}$ & $\begin{array}{l}34,60 \\
39,35\end{array}$ & $\begin{array}{l}15,4 \\
15,4\end{array}$ & $\begin{array}{l}28,5 \\
28,8\end{array}$ & $\begin{array}{l}375 \\
390\end{array}$ & & & $\begin{array}{l}375 \\
390\end{array}$ & $\begin{array}{l}0 \\
0\end{array}$ & $\begin{array}{l}0 \\
0\end{array}$ & $\begin{array}{l}88,5 \\
84\end{array}$ & $\begin{array}{l}63 \\
62\end{array}$ & $\begin{array}{l}41 \\
44\end{array}$ & $\begin{array}{l}69,5 \\
82\end{array}$ & $\begin{array}{l}3 \\
2\end{array}$ & $\begin{array}{l}584 \\
490\end{array}$ & $\begin{array}{l}181 \\
187\end{array}$ & $\begin{array}{l}32 \\
45\end{array}$ \\
\hline CIU & $\begin{array}{c}4 \\
5 \\
6 \\
7 \\
8 \\
9 \\
39 \\
40 \\
41 \\
42\end{array}$ & $\begin{array}{l}40,35 \\
40,45 \\
40,65 \\
40,25 \\
40,75 \\
40,95 \\
39,35 \\
39,45 \\
39,6 \\
39,55\end{array}$ & $\begin{array}{l}15,4 \\
15,5 \\
15,3 \\
15,5 \\
15,4 \\
15,6 \\
15,7 \\
15,7 \\
15,2 \\
15,4\end{array}$ & $\begin{array}{l}28,8 \\
28,5 \\
29,1 \\
29,9 \\
28,9 \\
28,6 \\
28,5 \\
28,6 \\
28,3 \\
28,6\end{array}$ & $\begin{array}{l}215 \\
220 \\
208 \\
190 \\
210 \\
165 \\
125 \\
130 \\
130 \\
135\end{array}$ & $\begin{array}{l}3,35 \\
3,4 \\
3,25 \\
3,25\end{array}$ & $\begin{array}{l}1,65 \\
1,7 \\
1,05 \\
1,8\end{array}$ & $\begin{array}{r}515 \\
170 \\
308 \\
790 \\
410 \\
565 \\
300 \\
470 \\
50 \\
150\end{array}$ & $\begin{array}{c}2,45 \\
\\
4,6 \\
1.15 \\
2,65 \\
-1,1 \\
0 \\
-4,95 \\
-3\end{array}$ & $\begin{array}{c}1,3 \\
\\
2,75 \\
0,8 \\
-0,4 \\
0 \\
-2,5 \\
-1,65\end{array}$ & $\begin{array}{l}74 \\
20 \\
82 \\
75 \\
50 \\
85 \\
58 \\
54 \\
33 \\
42,5\end{array}$ & $\begin{array}{l}54 \\
18,5 \\
62,5 \\
50,5 \\
38,5 \\
58,5 \\
43 \\
42,5 \\
24 \\
30\end{array}$ & $\begin{array}{l}39 \\
15 \\
46 \\
35 \\
28,5 \\
40 \\
27 \\
30 \\
17 \\
18,5\end{array}$ & $\begin{array}{l}61,5 \\
16,5 \\
66 \\
47 \\
36 \\
60,5 \\
57,5 \\
47,5 \\
22,5 \\
45\end{array}$ & $\begin{array}{l}1,85 \\
2,9 \\
2 \\
2,5 \\
2,3 \\
1,4 \\
1,75 \\
1,65 \\
2,45 \\
1,95\end{array}$ & $\begin{array}{l}454 \\
274 \\
501 \\
538 \\
412 \\
450 \\
288 \\
360 \\
258 \\
199\end{array}$ & $\begin{array}{r}193 \\
19 \\
121 \\
264 \\
186 \\
244 \\
114 \\
197 \\
35 \\
71\end{array}$ & $\begin{array}{l}55 \\
30 \\
21 \\
26 \\
45 \\
20 \\
38 \\
52 \\
62 \\
18\end{array}$ \\
\hline EIU & $\begin{array}{l}16 \\
17 \\
18 \\
21 \\
44 \\
47 \\
50\end{array}$ & $\begin{array}{l}40,15 \\
40,35 \\
40,25 \\
41,05 \\
39,05 \\
39,25 \\
39,85\end{array}$ & $\begin{array}{l}15,6 \\
15,8 \\
15,9 \\
15,7 \\
15,5 \\
15,5 \\
15,5\end{array}$ & $\begin{array}{l}28,4 \\
28,7 \\
27,3 \\
27,8 \\
28,6 \\
28,9 \\
28,7\end{array}$ & $\begin{array}{l}130 \\
160 \\
155 \\
175 \\
120 \\
110 \\
140\end{array}$ & $\begin{array}{l}3,7 \\
4,05 \\
3,9\end{array}$ & $\begin{array}{l}1,35 \\
1,35 \\
1,3\end{array}$ & $\begin{array}{l}430 \\
560 \\
755 \\
200 \\
150 \\
300 \\
150\end{array}$ & $\begin{array}{c}3,95 \\
4,35 \\
4,35 \\
1 \\
-2,3 \\
-0,96 \\
-3,1\end{array}$ & $\begin{array}{c}2,15 \\
2,5 \\
2,05 \\
- \\
- \\
-0,5 \\
-1,15\end{array}$ & $\begin{array}{l}60,5 \\
85 \\
88 \\
45 \\
50 \\
22,5 \\
20\end{array}$ & $\begin{array}{l}43,5 \\
55 \\
55 \\
32,5 \\
31 \\
22 \\
16,5\end{array}$ & $\begin{array}{l}31 \\
35.5 \\
34 \\
22 \\
20 \\
18 \\
12\end{array}$ & $\begin{array}{l}44 \\
49 \\
74,5 \\
39 \\
46 \\
16\end{array}$ & $\begin{array}{l}-2,1 \\
-3,2 \\
-3 \\
-3,3 \\
-2,2 \\
-3,1 \\
-2,7\end{array}$ & $\begin{array}{l}-422 \\
-590 \\
-478 \\
-265 \\
-256 \\
-436 \\
-166\end{array}$ & $\begin{array}{l}-267 \\
-347 \\
-140 \\
-100 \\
-110 \\
-308 \\
-84\end{array}$ & $\begin{array}{l}11 \\
31 \\
21 \\
28 \\
16\end{array}$ \\
\hline
\end{tabular}

TABLEAUIV Essais triaxiaux drainés (CID, EID). Paramètres d'état des éprouvettes et principaux résultats d'essais. Drained triaxial tests (CID, EID). State parameters of the specimens and main test results.

\begin{tabular}{|c|c|c|c|c|c|c|c|c|c|c|c|c|c|c|c|c|c|}
\hline \multirow{2}{*}{\multicolumn{2}{|c|}{ Essai }} & \multicolumn{4}{|c|}{ Éprouvette } & \multirow{2}{*}{\multicolumn{2}{|c|}{$\begin{array}{c}\text { Reconsolidation } \\
\text { sous } \\
\sigma_{c}^{\prime}=470 \mathrm{KPa}\end{array}$}} & \multirow{2}{*}{\multicolumn{3}{|c|}{$\begin{array}{l}\text { Consolidation } \\
\text { sous } \sigma^{\prime}\end{array}$}} & \multicolumn{7}{|c|}{ Cisaillement } \\
\hline & & \multirow{2}{*}{$\begin{array}{c}\text { Proiniter } \\
z \\
(\mathrm{~m})\end{array}$} & \multicolumn{3}{|c|}{ Eirintal } & & & & & & \multicolumn{4}{|c|}{ Moluis dedifumarain } & \multicolumn{3}{|c|}{ Tequite } \\
\hline Type & $N^{\circ}$ & & $\begin{array}{c}\gamma_{d} \\
\left(\mathrm{kN} / \mathrm{m}^{3}\right)\end{array}$ & $\begin{array}{l}\text { W } \\
(\%)\end{array}$ & $\begin{array}{l}\sigma_{\phi p} \\
(\mathrm{kPa})\end{array}$ & $\begin{array}{l}\xi_{0} \\
(\%)\end{array}$ & $\begin{array}{l}\varepsilon_{m o} \\
(\%)\end{array}$ & $\begin{array}{c}\sigma_{s}^{\prime} \\
(\mathrm{kPa})\end{array}$ & $\begin{array}{l}\varepsilon_{v e} \\
(\%)\end{array}$ & $\begin{array}{l}\varepsilon_{\infty} \\
(\%)\end{array}$ & $\begin{array}{c}E_{k}^{\prime} \\
(\mathrm{MPa})\end{array}$ & $\begin{array}{c}E_{0 s}^{\prime} \\
\text { (MPa) }\end{array}$ & $\begin{array}{c}E_{7}^{\prime} \\
(\mathrm{MPa})\end{array}$ & $\begin{array}{c}E_{\%}^{\prime} \\
(\mathrm{MPa})\end{array}$ & $\begin{array}{l}\varepsilon_{x} \\
(\%)\end{array}$ & $\left(\begin{array}{c}(\sigma-\sigma)_{t} \\
(\mathrm{kPa})\end{array}\right.$ & $\alpha$ \\
\hline CID & $\begin{array}{l}10 \\
11 \\
12 \\
13 \\
14 \\
15 \\
23 \\
24 \\
25 \\
26 \\
29 \\
30 \\
33 \\
35 \\
36\end{array}$ & $\begin{array}{l}41,35 \\
41,45 \\
41,55 \\
41,65 \\
42,05 \\
42,15 \\
41,45 \\
41,55 \\
41,65 \\
41,75 \\
41,85 \\
41,95 \\
42,05 \\
42,15 \\
42,25\end{array}$ & $\begin{array}{l}15,6 \\
15,7 \\
15,7 \\
15,7 \\
15,5 \\
15,7 \\
15,5 \\
15,8 \\
15,5 \\
15,5 \\
15,6 \\
15,7 \\
16 \\
16 \\
16,3\end{array}$ & $\begin{array}{l}28,6 \\
28,5 \\
27,9 \\
27,8 \\
27,7 \\
27,7 \\
28,6 \\
28,2 \\
28,6 \\
28 \\
28,5 \\
26,8 \\
27,2 \\
26,8 \\
27,5 \\
\end{array}$ & $\begin{array}{l}135 \\
120 \\
125 \\
140 \\
145 \\
150 \\
150 \\
140 \\
155 \\
160 \\
135 \\
130 \\
155 \\
120 \\
145\end{array}$ & \begin{tabular}{l|l} 
& 3,7 \\
3,85 \\
3,5 \\
3,75 \\
3,65
\end{tabular} & $\begin{array}{l}1,05 \\
1,4 \\
1,7 \\
1,4\end{array}$ & $\begin{array}{r}335 \\
720 \\
425 \\
540 \\
195 \\
250 \\
750 \\
540 \\
455 \\
360 \\
150 \\
50 \\
470 \\
150 \\
50\end{array}$ & $\begin{array}{c}1,95 \\
6,85 \\
3,9 \\
3,85 \\
0,85 \\
1,3 \\
4,7 \\
3,45 \\
2 \\
0,45 \\
-2,9 \\
-6,7 \\
0 \\
-2,75 \\
-6,75\end{array}$ & $\begin{array}{c}1,15 \\
2,4 \\
1,35 \\
1,9 \\
0,2 \\
0,8 \\
2 \\
1,55 \\
0,85 \\
0,25 \\
-1,35 \\
0 \\
-1,4 \\
-1,95\end{array}$ & $\begin{array}{l}66,5 \\
62,5 \\
42 \\
52,5 \\
42 \\
55,5 \\
59 \\
68,5 \\
61 \\
26,5 \\
47,5 \\
27 \\
49,5 \\
65,5 \\
21\end{array}$ & $\begin{array}{l}30 \\
41,5 \\
32 \\
35 \\
34 \\
33 \\
41 \\
46 \\
40 \\
19 \\
22 \\
16,5 \\
31 \\
35 \\
9,5\end{array}$ & $\begin{array}{l}25 \\
34 \\
24 \\
26 \\
29 \\
28 \\
34 \\
34 \\
32 \\
16 \\
15 \\
125 \\
23 \\
25 \\
8\end{array}$ & $\begin{array}{l}22,8 \\
31,4 \\
22,1 \\
24,6 \\
27,5 \\
27,9 \\
32 \\
29,3 \\
28 \\
16,8 \\
30,7 \\
15,8 \\
18 \\
24,9 \\
7,7\end{array}$ & $\begin{array}{l}4,05 \\
4,85 \\
4,80 \\
4,35 \\
4,30 \\
3,90 \\
3,85 \\
4,55 \\
5,05 \\
3,35 \\
1,35 \\
3,70 \\
8,35 \\
3,60 \\
3,60\end{array}$ & $\begin{array}{r}870 \\
1206 \\
820 \\
778 \\
904 \\
764 \\
986 \\
990 \\
800 \\
286 \\
172 \\
189 \\
698 \\
632 \\
159\end{array}$ & $\begin{array}{l}22 \\
51 \\
59 \\
34 \\
20 \\
46 \\
55 \\
55 \\
41 \\
39 \\
58 \\
34 \\
62 \\
50 \\
57\end{array}$ \\
\hline EID & $\begin{array}{l}22 \\
27 \\
28 \\
31 \\
34 \\
37\end{array}$ & $\begin{array}{l}41,45 \\
41,55 \\
41,65 \\
41,95 \\
42,15 \\
42,25\end{array}$ & $\begin{array}{l}15,9 \\
16,2 \\
15,9 \\
15,9 \\
15,4 \\
15,6\end{array}$ & $\begin{array}{l}27,1 \\
26,5 \\
26,9 \\
27,3 \\
28,1 \\
28,5\end{array}$ & $\begin{array}{l}150 \\
160 \\
110 \\
105 \\
160 \\
120\end{array}$ & $\begin{array}{l}3,6 \\
2,45 \\
2,7 \\
3,35\end{array}$ & $\begin{array}{l}1,3 \\
1,65 \\
1,1 \\
1,6\end{array}$ & $\begin{array}{l}750 \\
560 \\
300 \\
150 \\
470 \\
150\end{array}$ & $\begin{array}{c}4,45 \\
2,8 \\
-0,65 \\
-3 \\
0 \\
-2,65\end{array}$ & $\begin{array}{c}1,8 \\
1,25 \\
-0,25 \\
-1,05 \\
0 \\
-1,3\end{array}$ & $\begin{array}{r}150 \\
62 \\
36 \\
69 \\
100 \\
27\end{array}$ & $\begin{array}{l}54,5 \\
42,5 \\
22 \\
16 \\
49,5 \\
15\end{array}$ & $\begin{array}{r}35 \\
32 \\
17 \\
9 \\
31 \\
10\end{array}$ & $\begin{array}{l}76,1 \\
41,7 \\
20,5 \\
49,8 \\
74,7 \\
11,8\end{array}$ & $\begin{array}{l}-3,2 \\
-3,05 \\
-4,00 \\
-2,05 \\
-2 \\
-3,25\end{array}$ & $\begin{array}{l}-426 \\
-434 \\
-270 \\
-100 \\
-373 \\
-139\end{array}$ & $\begin{array}{r}31 \\
20 \\
8 \\
21 \\
34 \\
21\end{array}$ \\
\hline
\end{tabular}




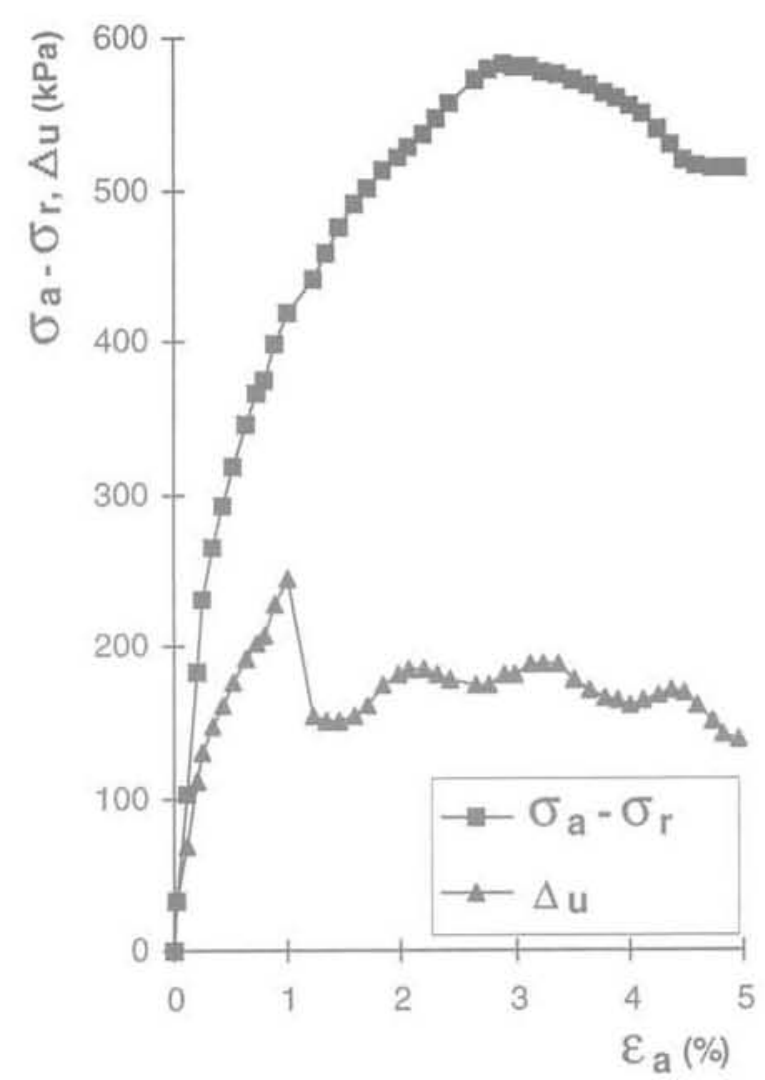

FG.1 Variations du déviateur et de la pression interstitielle en fonction de la déformation axiale dans le cas d'un essai UU (essai 1). Deviator stress and excess pore pressure versus axial strain for a specific UU test (test 1).

\section{$\varepsilon_{\mathrm{a}}(\%)$}

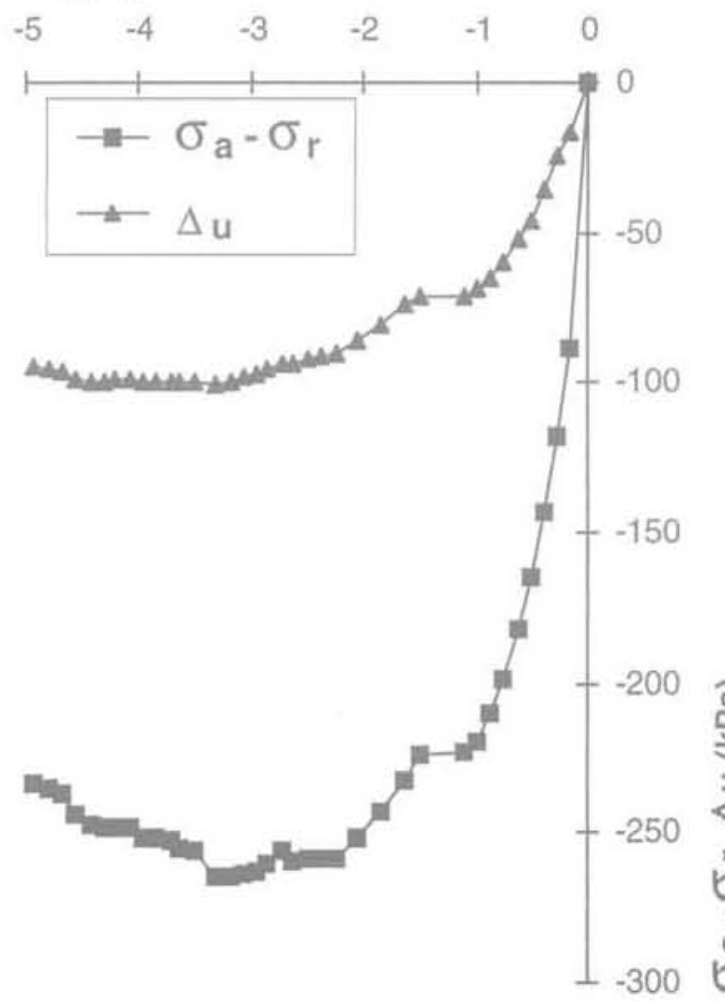

EG. 3 Variations du déviateur et de la pression interstitielle en fonction de la déformation axiale dans le cas d'un essai EIU (essai 21). Deviator stress and excess pore pressure versus axial strain for a specific EIU test (test 21).

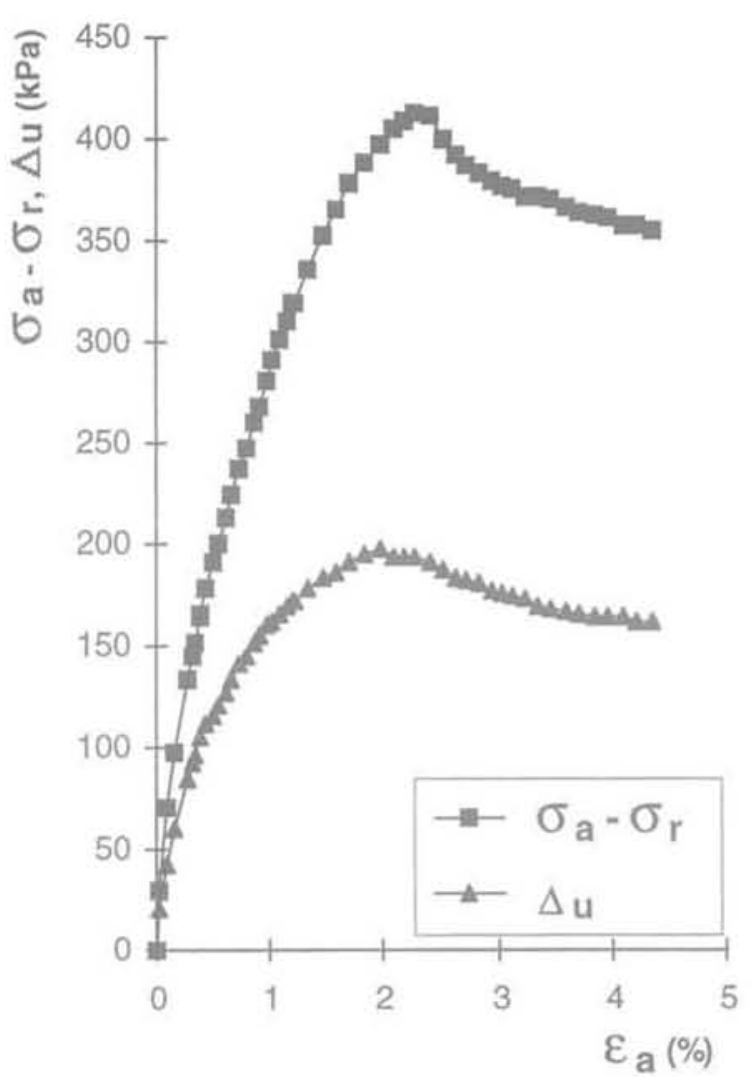

FlG.2 Variations du déviateur et de la pression interstitielle en fonction de la déformation axiale dans le cas d'un essai CIU (essai 8). Deviator stress and excess pore pressure versus axial strain for a specific CIU test (test 8 ).

met en évidence la « déconsolidation » du sol, c'est-àdire son remaniement progressif dans le temps. C'est à cause de ce remaniement que l'on n'a exécuté que deux essais UU et que, dans la deuxième partie de la campagne d'essais, les éprouvettes ont été systématiquement reconsolidées sous une contrainte isotrope de l'ordre de $\sigma_{\text {v. }}^{\prime}$. On notera que, le coefficient de pression du sol au repos $\mathrm{K}_{0}$ étant peu différent de l'unité (voir §4), cette reconsolidation peut être assimilée à une reconsolidation aux contraintes en place. La déformation volumique correspondante, comprise entre $2,45 \%$ et $4 \%$, est en moyenne de $3,45 \%$. Si, faute de disposer d'autre critère de remaniement des éprouvettes, on se réfère à celui établi par Berre (1985, cité par Lacasse et Berre, 1988) pour les éprouvettes d'argile sensible (tableau V), il apparaît que les éprouvettes déconsolidées (c'est-à-dire la quasi-totalité des éprouvettes triaxiales à l'exception de celles utilisées pour la réalisation des essais UU) se classent dans la catégorie «passable $»$.

TABLEAUV Critère de qualité proposé pour les éprouvettes d'argile sensible (d'après Berre, 1985).

Quality criterium suggested for sensitive clay specimens (after Berre, 1985).

\begin{tabular}{|c|l|}
\hline$\varepsilon .(\%)$ & \multicolumn{1}{|c}{ Qualitẻ de léprouvette } \\
\hline$<1$ & très bonne à excellente \\
$1-2$ & bonne \\
$2-4$ & passable \\
$4-8$ & mauvaise \\
$>8$ & très mauvaise \\
\hline
\end{tabular}



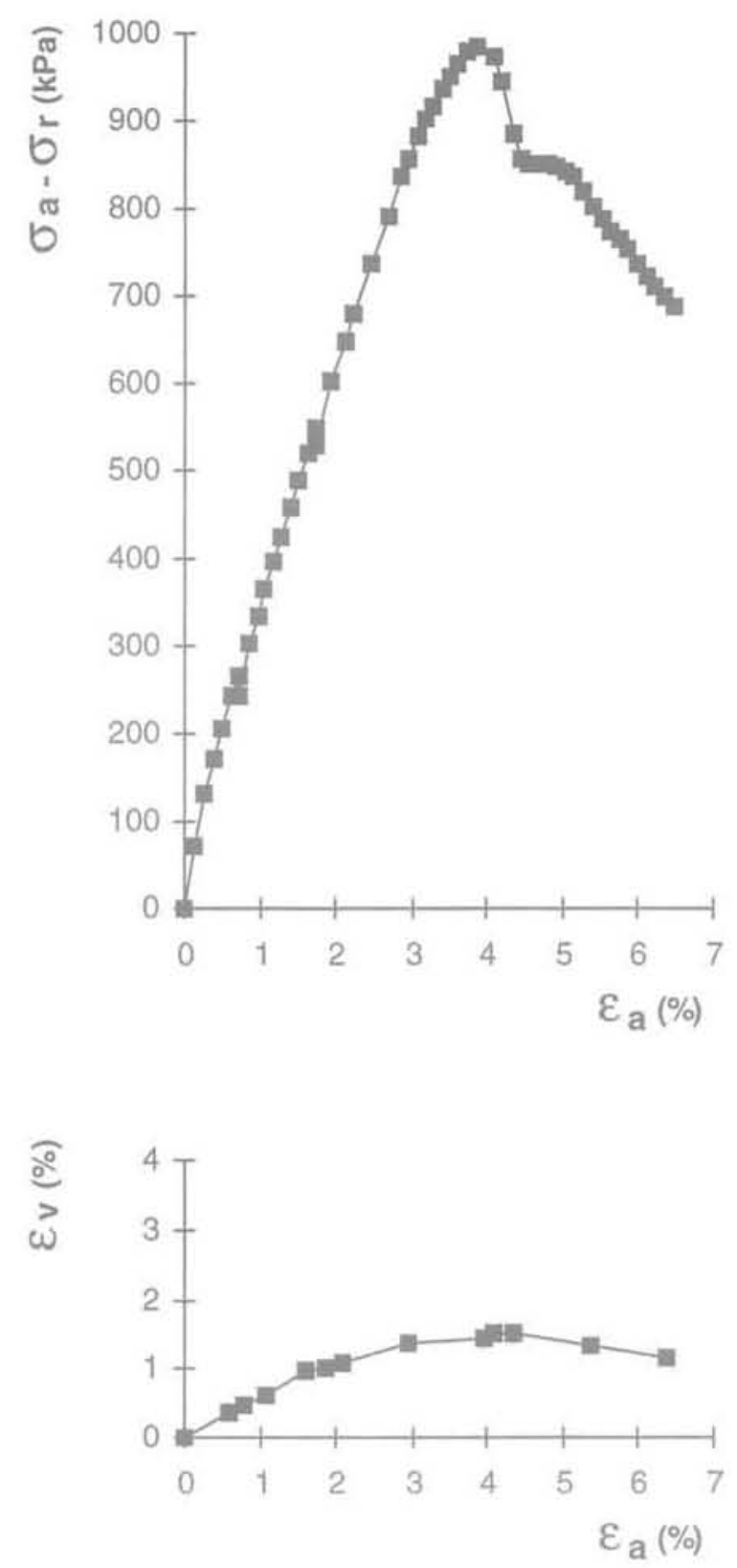

Fig,4 Variations du déviateur et de la déformation volumique en fonction de la déformation axiale dans le cas d'un essai CID (essai 23).

Deviator stress and volumetric strain versus axial strain for a specific CID test (test 23).

Les courbes déviateur-déformation axiale obtenues pour la grande majorité des essais, sont caractérisées par un pic plus ou moins accusé, qui est atteint pour une valeur $\varepsilon_{\text {af }}$ de la déformation axiale $\varepsilon_{\mathrm{a}}$ généralement comprise entre $1,5 \%$ et $5 \%$ (entre $1,4 \%$ et $3 \%$ dans le cas des essais UU et CIU, entre $-3,3 \%$ et $-2,1 \%$ dans le cas des essais EIU, entre $3,05 \%$ et $5,05 \%$ dans le cas des essais CID et entre $-4 \%$ et $-2 \%$ dans le cas des essais EID). Ce pic marque la rupture des éprouvettes qui se manifeste par la formation d'un plan de glissement dont l'inclinaison $\alpha$ sur l'horizontale varie dans de larges limites. Dans quelques cas, on note également l'apparition d'un second plan de rupture, souvent subhorizontal.

Les valeurs du déviateur ont été calculées sans tenir compte des efforts parasites introduits par la membrane et le filtre latéral. Avant l'apparition du plan de glisse-

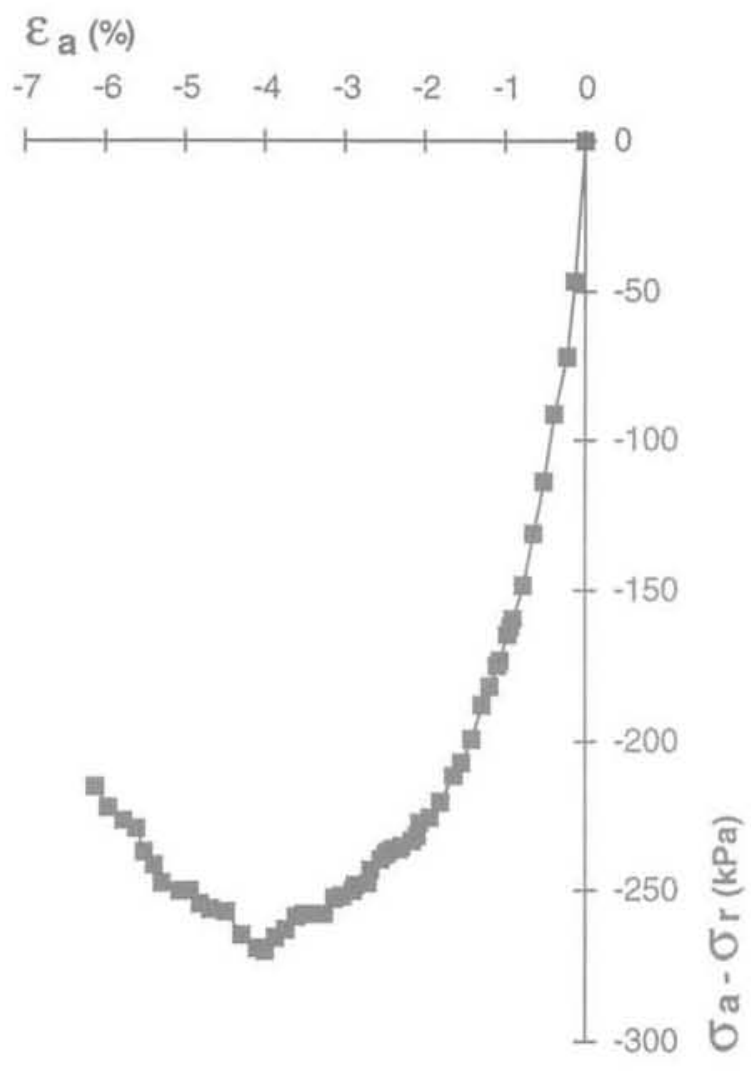

\section{$\varepsilon_{\mathrm{a}}(\%)$}

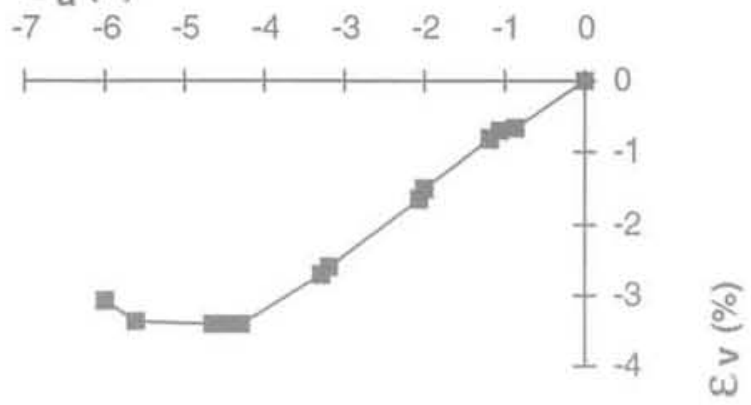

月6.5 Variations du déviateur et de la déformation volumique en fonction de la déformation axiale dans le cas d'un essai EID (essaỉ 28).

Deviator stress and volumetric strain versus axial strain for a specific EID test (test 28).

ment, c'est-à-dire avant le déviateur atteigne sa valeur de pic $\left(\sigma_{\mathrm{a}}-\sigma_{\mathrm{p}}\right)_{\mathrm{p}}$ ces efforts sont négligeables devant le frottement du piston en tête de cellule. D'après Webb (1969), ce frottement, mesuré au cours d'essais sur l'argile de Londres effectués au moyen d'un équipement semblable à celui utilisé pour les essais sur l'argile des Flandres, peut atteindre $2 \%$ à $8 \%$ de la force axiale exercée par la presse avant la rupture et $2 \%$ à $3 \%$ lors de la rupture. Après la formation du plan de rupture, le glissement relatif des deux parties de l'éprouvette provoque une forte augmentation des efforts repris par la membrane, un accroissement du frottement du piston ainsi que le développement d'une force horizontale dans le plan de rupture. Il s'ensuit que les valeurs absolues du déviateur sont surestimées de $2 \%$ à $8 \%$ avant et jusqu'à la rupture, qu'elles ne sont pas représentatives du comportement du sol après la rupture et qu'elles le 


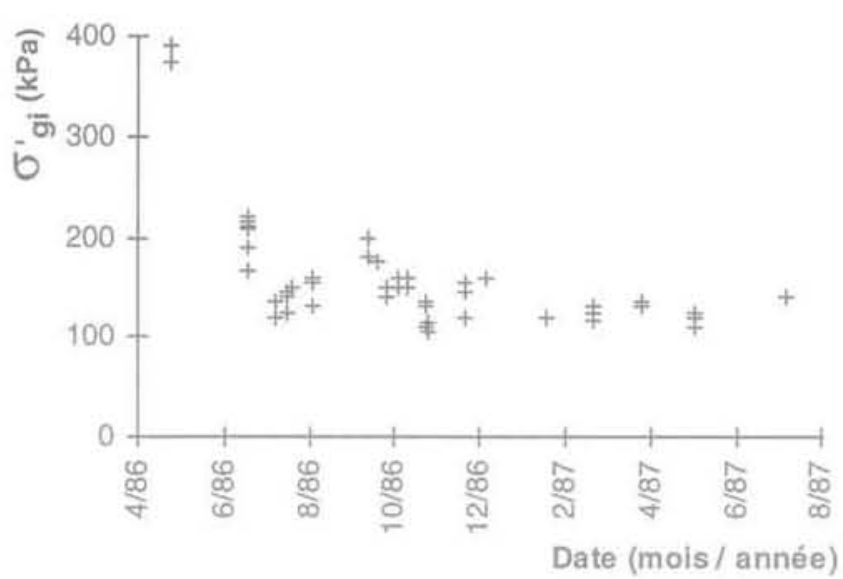

FG. 6 Variations de la contrainte isotrope de gonflement avec le temps. Les deux premières mesures ont été réalisées environ 5 mois après le prélèvement de I'argile.

Swelling pressure versus time. The first two measurements were carried out about five months after the sampling of the clay.

sont d'autant moins que la valeur absolue de la différence $\varepsilon_{\mathrm{a}}-\varepsilon_{\mathrm{af}}$ est plus grande.

L'erreur par excès commise sur le déviateur explique, au moins en partie, que, dans le cas de cinq essais EIU (dont les résultats n'ont pas été retenus), le calcul ait indiqué des valeurs négatives de la contrainte axiale effective $\sigma^{\prime}$ lorsque la force axiale mesurée s'approchait de sa valeur maximale.

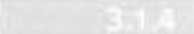

\section{Essais au cône de laboratoire}

Des essais au cône de laboratoire (fall cone) ont été réalisés sur des éprouvettes préalablement consolidèes dans des cellules œedométriques. Ils avaient pour objectif de déterminer la loi qui régit les variations de la cohésion non drainée lors du déchargement de l'argile. Ces essais n'étaient pas a priori les mieux adaptés à l'étude de la résistance au cisaillement d'une argile raide dans laquelle le cône ne pénètre que peu profondément. Ils ont cependant été retenus car, intéressant un faible volume de sol, ils permettent de s'affranchir de la dispersion due à la fissuration qui affecte des essais triaxiaux. En outre, de nombreux essais peuvent être pratiqués sur une même éprouvette à divers stade de sa consolidation, ce qui limite la dispersion liée à l'hétérogénéité du sol.

Les essais ont èté effectués au moyen d'un cône d'angle $30^{\circ}$ et de masse $400 \mathrm{~g}$. Chaque éprouvette était montée dans une cellule codométrique puis consolidée sous une contrainte verticale $\sigma^{\prime}$. La cellule était ensuite retirée du bâti œdométrique et neuf essais de poinçonnement étaient effectués sur la face inférieure de l'éprouvette (un au centre, les huit autres uniformément répartis sur une circonférence de même centre que l'éprouvette et de rayon moitié). La cellule était alors remontée sur le bâti et l'éprouvette reconsolidée sous une contrainte verticale $\sigma_{v}^{\prime}$ inférieure à $\sigma_{\text {y. }}^{\prime}$. A la fin de cette reconsolidation, l'éprouvette était à nouveau déchargée et neuf essais de poinçonnement exécutés sur sa face supérieure. Deux séries d'éprouvettes ont été essayées suivant cette procédure :
- dans le cas de la première série, on a affecté à $\sigma_{\mathrm{vr}}^{\prime}$ la valeur $500 \mathrm{kPa}$, c'est-à-dire une valeur de l'ordre de la contrainte verticale effective en place, $\sigma^{\prime}$ étant comprise entre $1 \mathrm{kPa}$ et $250 \mathrm{kPa}$;

- les éprouvettes de la deuxième série ont été consolidées sous $\sigma_{y=}^{\prime}=3000 \mathrm{kPa}$, c'est-à-dire sous une contrainte de l'ordre de la pression de préconsolidation de l'argile, les valeurs de $\sigma_{y}^{\prime}$ étant comprises entre $1 \mathrm{kPa}$ et $500 \mathrm{kPa}$.

Les essais ont été interprétés à partir de la relation :

$$
c_{u}=K \frac{Q}{h^{2}}
$$

dans laquelle :

h désigne l'enfoncement du cône,

Q la masse du cône,

$\mathrm{K}$ un coefficient adimensionnel fonction de l'angle du cône et, dans une moindre mesure, de la nature de l'argile.

La valeur de K n'étant pas connue, it n'était pas possible de déterminer les valeurs de la cohésion non drainée qui correspondent aux différentes pressions de consolidation. En revanche, $\mathrm{K}$ étant constant pour une même argile, il était possible de déterminer pour chacune des éprouvettes, dans la mesure où elles sont homogènes, le rapport de la cohésion $\mathrm{c}_{\mathrm{u}}$ de l'argile reconsolidée sous $\sigma_{\mathrm{v}}^{\prime}$ et de la cohésion $c_{\mathrm{u}}^{\sigma^{\prime} v r}$ de l'argile consolidée sous $\sigma_{* \psi^{\prime}}^{\prime}$. Celui-ci est donné par l'expression:

$$
\frac{c_{u}}{c_{u}^{\sigma_{v r}}}=\left(\frac{h_{r}}{h}\right)^{2}
$$

dans laquelle $h$, et $h$ représentent respectivement les valeurs de l'enfoncement du cône après consolidation de l'argile sous $\sigma_{\mathrm{vt}}^{\prime}$ et après reconsolidation sous $\sigma_{\mathrm{y}}^{\prime}$ Les valeurs moyennes de $h$, et $h$ relatives à chaque éprouvette, ainsi que les valeurs correspondantes du rapport $\frac{C_{u}}{c_{u}^{\sigma_{u}}}$ sont présentées dans le tableau VI.

\section{2}

\section{Études réalisées lors de la reconnaissance des sites d'ouvrages}

Les campagnes de reconnaissance des sites du QPO à Dunkerque et du QEP à Calais ont comporté l'exécution d'une importante quantité d'essais de laboratoire et d'essais en place. Nombre d'entre eux fournissent des données qui n'ont pu être obtenues à partir de l'étude en laboratoire décrite précédemment et qui permettent de compléter et de préciser ses résultats. Les résultats de ces essais, dont le type, le nombre et les principales conditions d'exécution sont indiqués succinctement ci-après, ont donc été pris en compte pour la détermination des propriétés mécaniques de l'argile.

\section{4}

\section{Essais de laboratoire}

Ils ont été effectués au LRPC de Lille sur des échantillons prélevés à Dunkerque et à Calais dans les 20 mètres supérieurs de la couche d'argile. On a retenu 
TABLEAUVI Essais au cône de laboratoire. Paramètres d'état des éprouvettes et résultats d'essais. Fall-cone tests. State parameters of the specimens and test results.

\begin{tabular}{|c|c|c|c|c|c|c|}
\hline \multirow[t]{2}{*}{$\gamma_{d}\left(\mathrm{kN} / \mathrm{m}^{3}\right)$} & \multirow[t]{2}{*}{ w\% } & \multirow[t]{2}{*}{$\sigma_{\mathrm{w}}^{\prime}(\mathrm{kPa})$} & \multirow[t]{2}{*}{$\sigma_{V}(\mathrm{kPa})$} & \multicolumn{2}{|c|}{$\begin{array}{l}\text { Profondeur moyenne } \\
\text { de pénétration ( } \mathrm{mm} \text { ) }\end{array}$} & \multirow{2}{*}{$\frac{c_{u}}{c_{u}^{\sigma}{ }_{u r}}$} \\
\hline & & & & h. & $\mathrm{h}$ & \\
\hline 15,4 & 28,3 & & 1 & 2,27 & 5 & 0,206 \\
\hline 15,1 & 28,2 & 500 & 30 & 2,30 & 4,02 & 0.327 \\
\hline 15 & 29,5 & & 60 & 2,66 & 3,8 & 0,49 \\
\hline 14.9 & 31.8 & & 1 & 2,39 & 6,47 & 0,136 \\
\hline 15,2 & 29,3 & & 30 & 2,32 & 4,27 & 0,295 \\
\hline 15,7 & 27,3 & 3000 & 60 & 2,14 & 3,62 & 0,349 \\
\hline 15,6 & 28 & & 120 & 2,28 & 3,62 & 0,396 \\
\hline 15,3 & 29,1 & & 250 & 2,44 & 3,21 & 0,577 \\
\hline 15,3 & 28,8 & & 500 & 2,46 & 2,85 & 0,745 \\
\hline
\end{tabular}

les résultats des essais triaxiaux et de cisaillement direct de type UU, ainsi que les résultats des essais de cisaillement direct de type $C D$ :

- les essais triaxiaux UU ont été réalisés sur les échantillons prélevés dans les deux sites : 34 éprouvettes en provenance de Dunkerque et 42 éprouvettes en provenance de Calais ont été essayées (vitesse de déformation $1.1 \mathrm{~mm} / \mathrm{min}$ ). La plupart des éprouvettes avaient un diamètre de $35,6 \mathrm{~mm}$ et une hauteur de $80 \mathrm{~mm}$ et étaient taillées dans le sens vertical. Cependant, les dimensions de 6 éprouvettes du premier site étaient respectivement de $76 \mathrm{~mm}$ et de $160 \mathrm{~mm}$ tandis que 9 éprouvettes du second avaient été découpées dans le sens horizontal :

- les essais de cisaillement direct UU ont été réalisés sur 4 éprouvettes découpées dans l'argile de Dunkerque (vitesse de déformation $1,2 \mathrm{~mm} / \mathrm{min}$ ) ;

- les essais de cisaillement direct CD ont mis en jeu 52 éprouvettes découpées dans l'argile de Calais (vitesse de déformation $8 \mu \mathrm{m} / \mathrm{min}$ ).

\section{3ingor}

\section{Essais en place}

Cinq types différents d'essais en place ont été exécutés dans l'argile sur les deux sites étudiés :

- des essais pressiométriques Ménard à Dunkerque et à Calais (cinq sondages à Dunkerque et un sondage à Calais):

- des essais au pressiomètre autoforeur à Dunkerque (un sondage) ;

- des essais scissométriques de chantier (un sondage);

- des essais de pénétration statique (un sondage) ;

- des essais au dilatomètre Marchetti (un sondage);

les trois dernières techniques d'essai, mises en cuvre à l'initiative de l'Institut Français du Pétrole (IFP, 1992), n'ayant intéressé que le site de Calais.

Les conditions d'exécution de certains essais ou leurs résultats appellent les commentaires suivants.

L'examen des courbes d'expansion obtenues à Dunkerque à partir des essais pressiométriques Ménard faisant apparaitre un resserrement des parois des forages, les résultats de ces essais ne peuvent être considérés comme fiables, aussi n'a-t-on tenu compte que des caractéristiques pressiométriques mesurées à Calais.

Les essais au pressiomètre autoforeur ont été réalisés depuis le fond d'une fouille de grande largeur de $12,5 \mathrm{~m}$ de profondeur, ouverte à l'emplacement du QPO lors de la construction de celui-ci, et maintenue hors d'eau par un rabattement local de la nappe du sable flandrien. Les travaux d'excavation de cette fouille se sont étagés sur trois mois et n'étaient terminés que depuis environ deux mois lors de l'exécution des essais, si bien qu'un processus de consolidation locale de l'argile n'avait pu s'amorcer dans la zone déchargée. Il s'ensuit que les variations de pression interstitielle, qui se sont produites dans cette zone lors des terrassements, résultent uniquement du déchargement de l'argile à volume constant et sont sans rapport avec le niveau de la nappe du sable après rabattement. En ce qui concerne le déroulement des essais, il est important de préciser que le temps écoulé entre la mise en place de la sonde et le début d'un essai n'a été que de 15 à 20 minutes. Cette durée était sans doute insuffisante pour que la pression exercée par la sonde à la fin de cette période de repos se soit stabilisée à la

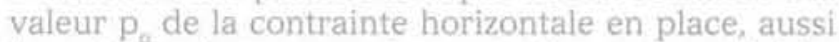
les valeurs de $\mathrm{p}_{\mathrm{o}}$ mesurées sont-elles vraisemblablement entachées d'une certaine erreur. Les essais au pressiomètre autoforeur ont également permis de déterminer la cohésion non drainée et le module de cisaillement de I'argile.

Les essais au dilatomètre Marchetti ont été réalisés par le LRPC de Blois (Boulinet et Waschkowski, 1994). Ils ont permis de déterminer en fonction de la profondeur (le pas des mesures était de $0,20 \mathrm{~m}$ ) les variations des paramètres attachés à ce type d'essai et notamment l'indice de contrainte horizontale $K_{D}$ qui sera utilisé dans ce qui suit.

Les essais de pénétration statique ont été effectués au moyen d'un piézocône. Ils ont fourni les valeurs q, de la résistance de pointe corrigée, qui prennent en compte les surpressions interstitielles mesurées au cours de la pénétration. 


\section{État des contraintes horizontales au repos}

La diversité des données expérimentales disponibles a permis d'évaluer les contraintes horizontales en place, c'est-à-dire le coefficient de pression du sol au repos, par quatre méthodes basées sur des principes entièrement différents. Le coefficient $\mathrm{K}_{0}$ a en effet été déterminé :

- à partir des mesures de pression horizontale totale au repos p effectuées au pressiomètre autoforeur ;

- à partir de l'indice de contrainte horizontale $\mathrm{K}_{D}$ mesuré au moyen du dilatomètre Marchetti ;

- à partir des deux valeurs apparemment fiables de la pression isotrope de gonflement $\sigma_{\text {gi }}^{\prime}$ mesurées à Dunkerque (cf. tableau III essais 1 et 3), interprétées par la méthode de Skempton (1961) ;

- à partir de la pression de préconsolidation $\sigma_{p}^{\prime}$ et de l'angle de frottement interne $\varphi^{\prime}$ de l'argile, au moyen d'une corrélation établie par Mayne et Kulhawy (1982).

\section{1}

\section{Interprétation des essais au pressiomètre autoforeur}

Les essais au pressiomètre autoforeur ont été réalisés depuis le fond d'une fouille de grande largeur de profondeur $\mathrm{z}_{\mathrm{F}}=12,5 \mathrm{~m}$. Comme indiqué précédemment (cf. § 3.2.2), le temps écoulé entre le début des travaux d'excavation et l'exécution de ces essais était de l'ordre de cinq mois, c'est-à-dire trop faible pour que la consolidation de l'argile ait pu s'amorcer dans la zone déchargée. Dans ces conditions, la sollicitation appliquée à l'argile est une sollicitation non drainée et la variation $\Delta u$ de pression interstitielle dans la zone déchargée ne dépend que de la variation des contraintes totales. Compte tenu de la largeur de la fouille, on peut considérer qu'à l'emplacement du sondage pressiométrique, les contraintes effectives initiales verticale $\sigma_{v 0}^{\prime}$ et horizontale $p^{\prime}=K_{0} \sigma_{y p}^{\prime}$ ne sont pas modifiées par l'excavation et que la variation de pression interstitielle est égale à la variation de contrainte verticale totale, soit :

$$
\Delta u=-\gamma z_{F}=-20 \times 12,5=-250 \mathrm{kPa}
$$

La pression interstitielle au niveau d'un essai est alors :

$$
u=u_{0}+\Delta u=u_{0}-\gamma z_{F}
$$

$u_{0}$ étant la pression interstitielle initiale définie par le niveau de la nappe du sable avant rabattement et terrassement.

La contrainte horizontale effective est alors évaluée à partir de la valeur $p_{0}$ mesurée au pressiomètre autoforeur, au moyen de la relation:

$$
p_{0}^{\prime}=p_{0}-u_{0}+\gamma z_{F}
$$

et on en déduit la valeur de $\mathrm{K}_{0}$ au niveau de l'essai, soit :

$$
K_{o}=\frac{p_{0}-u_{0}+\gamma z_{F}}{\sigma_{v o}^{\prime}}
$$

\section{Exploitation des essais au dilatomètre Marchetti}

La détermination de $\mathrm{K}_{0}$ à partir de l'indice de contrainte horizontale $\mathrm{K}_{\mathrm{D}}$ a été faite au moyen de la corrélation :

$$
K_{0}=\left[\frac{K_{D}}{1,5}\right]^{0,47}-0,6 \quad \text { (Marchetti , 1980) }
$$

\section{3}

\section{Application de la méthode de Skempton (1961)}

Skempton a montré qu'il est possible d'obtenir un ordre de grandeur de la valeur de $\mathrm{K}_{0}$ d'une argile raide saturée, à une profondeur donnée, à partir de la pression interstitielle négative initiale u mesurée sur un échantillon intact prélevé à cette profondeur. La pression interstitielle $u_{j}$, qui est égale au signe près à $\sigma_{\sigma,}^{\prime}$, peut en effet s'exprimer en fonction de $K_{\alpha}$ de la contrainte verticale effective en place $\sigma$ au niveau étudié et du coefficient de pression interstitielle $A_{\text {s }}$ mis en jeu lors du prélèvement

$$
u_{i}=-\sigma_{g i}^{\prime}=-\sigma_{v o}^{\prime}\left[K_{0}\left(1-A_{s}\right)+A_{s}\right]
$$

A étant de l'ordre de 0,3 dans le cas de l'argile de Londres.

L'évaluation de $K_{0}$ à partir de $\sigma_{g i}^{\prime}$ a été faite au moyen de la relation précédente en conservant la valeur $A_{s}=0,3$, ce que justifie la similitude des propriétés de l'argile des Flandres et de l'argile de Londres.

\section{4}

\section{Corrélation de Mayne et Kulhawy (1982)}

A chaque niveau d'une couche surconsolidée ayant une histoire analogue à celle de l'argile des Flandres (Fig, 7), c'est-à-dire ayant tout d'abord été chargée à la

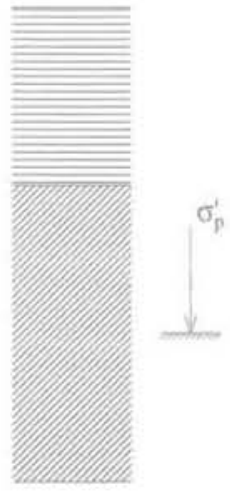

(a)
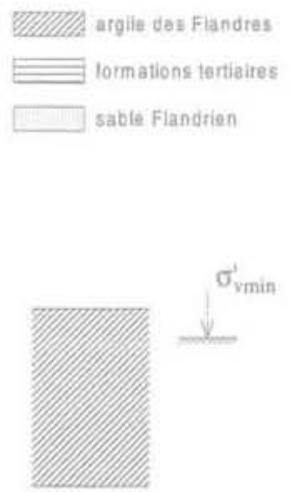

(b)

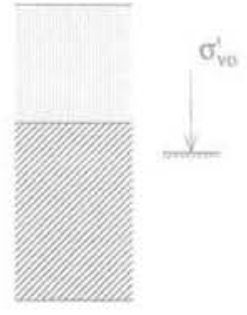

(c)
FG. 7 Schématisation de l'histoire de l'argile des Flandres :

a-déposition et chargement par des formations sus-jacentes,

b- déchargement consécutif à l'érosion de celles-ci,

c-rechargement par le sable flandrien.

Stress history of the Flanders clay:

a - deposition and loading by overlying layers,

$\mathrm{b}$ - unloading through erosion of these lavers, $\mathrm{c}$ - reloading by flandrian sand. 
contrainte $\sigma_{p}^{\prime}$ (déposition de sols sus-jacents), déchargée à la contrainte $\sigma_{y \text { min }}$ (érosion) puis rechargée à la contrainte $\sigma^{\prime}$ (reprise de sédimentation), le coefficient $\mathrm{K}_{\mathrm{o}}$ peut être évalué à partir de la corrélation de Mayne et Kulhawy :

$$
K_{o}=\left(1-\sin \varphi^{\prime}\right)\left[\frac{R_{o c}}{R_{o c \max }^{0-4 \max }}+\frac{3}{4}\left(1-\frac{R_{o c}}{R_{o c \max }}\right)\right]
$$

avec $R_{o c}=\frac{\sigma_{p}^{\prime}}{\sigma_{\mathrm{io}}^{\prime}}$ (rapport de surconsolidation actuel)

et $R_{o c \max }=\frac{\sigma_{p}}{\sigma_{v \text { min }}}$ (rapport de surconsolidation maximal).

$\mathrm{R}_{\mathrm{oc}}$ et $\mathrm{R}_{\mathrm{ocmax}}$ ont pour valeur limite

$$
R_{\mathrm{oc} \lim }=\left[\frac{1+\sin \varphi^{\prime}}{\left(1-\sin \varphi^{\prime}\right)^{2}}\right]^{\frac{1}{\sin \varphi}}
$$

Les variations de $\mathrm{K}$ avec la profondeur dans l'argile ont été déterminées au moyen de la relation précédente à partir des données numériques suivantes :

$$
\begin{aligned}
& \varphi^{\prime}=23^{\circ} \\
& \sigma_{p}^{\prime}=\sigma_{p}^{\left(x^{*}=0 \mid\right.}+\gamma z^{*}=3100+10 z^{*}
\end{aligned}
$$

$\sigma^{\prime}$ étant exprimé en kilopascals et $z^{*}$, exprimé en mêtres, représentant la profondeur dans l'argile.

\section{Résultats et discussion}

Les résultats obtenus sont reportés sur le diagramme de la figure 8 . On constate que les points expérimentaux obtenus à partir des essais au pressiomètre autoforeur, des essais au dilatomètre et des mesures de pression isotrope de gonflement sont groupés autour de la courbe de variation de $\mathrm{K}$ déterminée à partir de la corrélation de Mayne et Kulhawy, à l'exception de cinq d'entre eux. On remarquera que ces derniers ont été obtenus à partir de mesures au pressiomètre autoforeur dont la fiabilité est discutable en raison d'un temps de relaxation de la sonde sans doute trop court. Les trois points correspondant aux plus faibles valeurs de $\mathrm{K}$ sont manifestement aberrants, puisque ces valeurs sont de l'ordre de celle que l'on obtiendrait pour l'argile normalement consolidée. Les deux autres valeurs discordantes, les plus élevées, sont vraisemblablement liées à un phénomène de «bourrage $»$ qui se serait produit lors de la mise en place de la sonde aux niveaux considérés.

Il apparaît donc que les quatre méthodes appliquées à la détermination de $\mathrm{K}$ conduisent à des résultats relativement semblables, ceci bien qu'elles mettent en jeu des données physiques et des principes fondamentalement différents. Cette concordance constitue une forte présomption de la représentativité des résultats obtenus et l'on peut conclure que, sur les 12 mètres supérieurs de la couche, le coefficient de pression du sol au repos est vraisemblablement compris entre 0,9 et 1,1 .

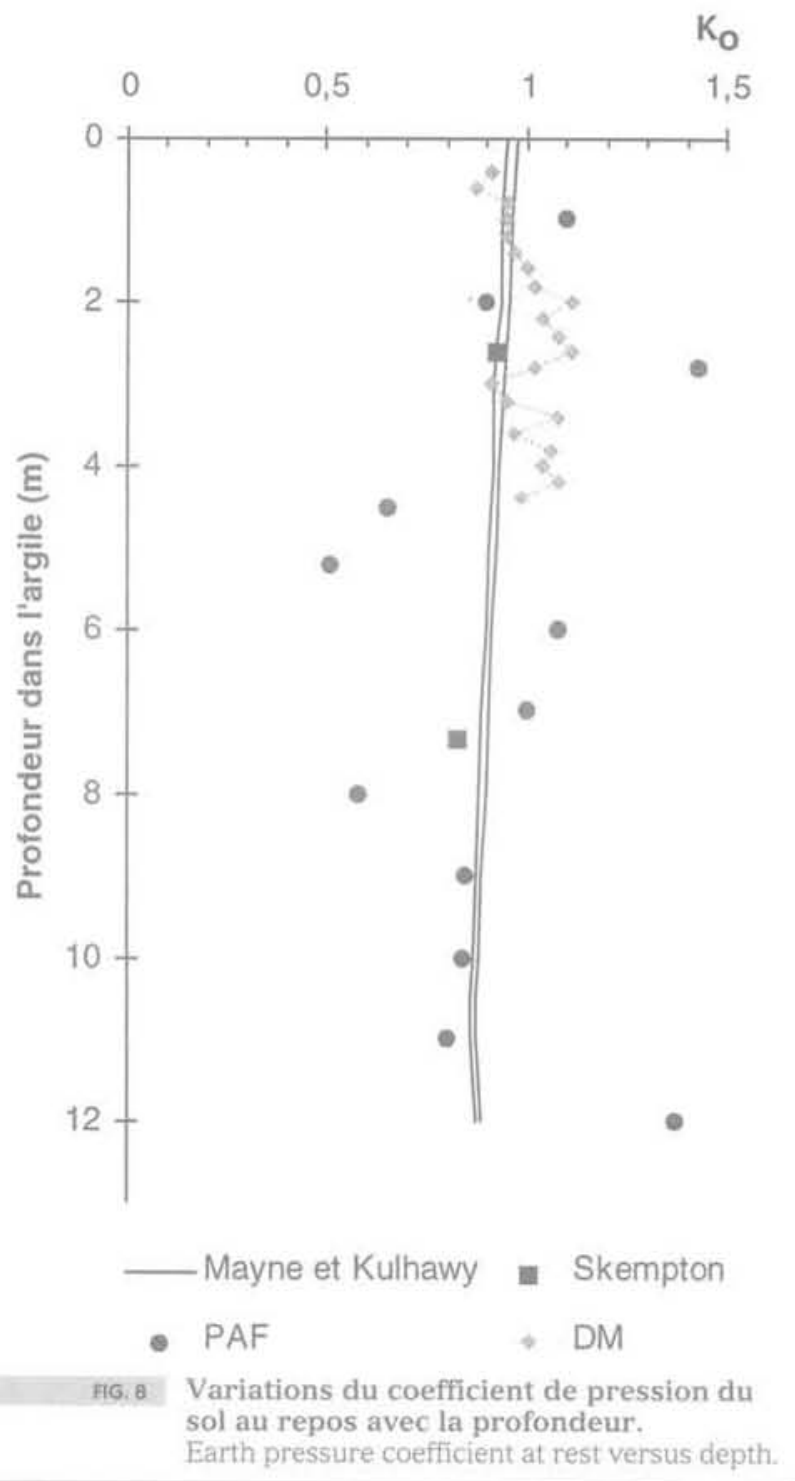

\section{5}

\section{Résistance au cisaillement non drainé}

\section{1}

\section{Généralités}

Les données relatives à la résistance au cisaillement non drainé tirées des essais triaxiaux réalisés au LCPC sont, dans l'ensemble, difficilement interprétables. En effet, d'une part, la grande majorité de ces essais ont été effectués sur des éprouvettes plus ou moins remaniées et, d'autre part, ces éprouvettes se sont rompues suivant des plans de glissement diversement inclinés. Or, lorsque l'inclinaison $\alpha$ du plan de rupture sur l'horizontale diffère sensiblement de $45^{\circ}$, la résistance mobilisée n'est pas égale à:

$$
t_{f}=\left(\frac{\sigma_{a}-\sigma_{r}}{2}\right)
$$


mais à $\tau_{f}=\left(\frac{\sigma_{u}-\sigma_{r}}{2}\right)_{f} \sin 2 a$.

Seuls les essais UU conduisent à des valeurs relativement fiables de $\mathrm{c}_{\mathrm{y}}$ dans la mesure où ils ont été effectués sur des éprouvettes peu remaniées (pour lesquelles $\sigma_{\text {u }}$ est égale à environ $90 \%$, de $\sigma_{\text {w }}^{\prime}$ c'est-à-dire à une valeur de l'ordre de la contrainte moyenne effective en place). Ces deux essais conduisent à des valeurs de $c_{u}$ respectivement égales à $262 \mathrm{kPa}\left(\mathrm{t}_{\mathrm{f}}=292 \mathrm{kPa}\right.$ et $\tau_{\text {f }}=$ $262 \mathrm{kPa})$ et à $245 \mathrm{kPa}\left(t_{r}=\tau_{f}=245 \mathrm{kPa}\right)$, correspondant à une valeur moyenne de l'ordre de $250 \mathrm{kPa}$.

Les résultats des essais CIU et EIU effectués sur des éprouvettes de moins bonne qualité ne permettent, en revanche, ni d'atteindre des valeurs suffisamment représentatives de la résistance non drainée, ni d'établir la loi de variation de $c_{\mathrm{u}}$ en fonction de $\sigma^{\prime}$, qui, dans le cas d'une argile fortement surconsolidée, détermine l'amplitude de la diminution de $\mathrm{c}_{4}$ associée à un déchargement de l'argile. L'examen du diagramme de la figure 9 , où sont reportées en fonction de $\sigma$ ' les valeurs $t$, et de $\tau$, obtenues à partir de l'ensemble des essais triaxiaux non drainés, montre, en effet, une forte dispersion des résultats, due au remaniement plus ou moins important des éprouvettes ainsi qu’à la fissuration de certaines d'entre elles.

On a donc étudié la loi de variation de c à partir des essais au cône de laboratoire décrits précédemment et l'on a cherché à déterminer la cohésion non drainée de l'argile en place à partir de l'ensemble des résultats des essais de laboratoire et essais en place effectués lors des différentes campagnes de reconnaissance des sites de Dunkerque et ce Calais (cf. \$ 3.2).

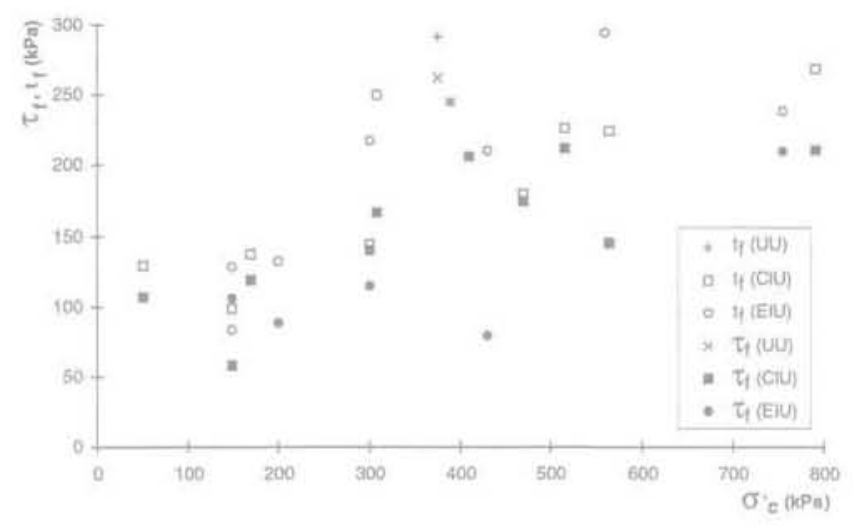

FG,9 Variations de la résistance au cisaillement non drainé avec la contrainte isotrope de consolidation.

Undrained shear strength versus isotropic consolidation stress.

\section{Variation de la cohésion non drainée avec la contrainte de consolidation dans le domaine surconsolidé}

La forme de la loi de variation de la cohésion non drainée $c_{4}$ d'une argile non fissurée consolidée, soit à déformation horizontale nulle sous une contrainte ver- ticale $\sigma^{\prime}$, soit sous une contrainte isotrope $\sigma^{\prime}$, découle des relations suivantes publiées par Mitachi et Kitago (1976) et par Ladd et al. (1977):

$$
\begin{aligned}
\frac{c_{u}}{\sigma_{v}^{\prime}} & =\frac{c_{u}^{n c}}{\sigma_{p}^{\prime}}\left(\frac{\sigma_{p}^{\prime}}{\sigma_{v}^{\prime}}\right)^{m} \\
\text { et } \frac{c_{u}}{\sigma_{c}^{\prime}} & =\frac{c_{u}^{n c i}}{\sigma_{p i}^{\prime}}\left(\frac{\sigma_{p i}^{\prime}}{\sigma_{c}^{\prime}}\right)^{m}
\end{aligned}
$$

dans lesquelles :

$\mathrm{C}_{u}^{n i c}$ représente la cohésion non drainée de l'argile normalement consolidée à déformation horizontale nulle sous la contrainte verticale $\sigma_{p}$;

$\mathrm{C}_{\mathrm{y}}^{\text {nci }}$ représente la cohésion non drainée de l'argile normalement consolidée sous la contrainte isotrope $\sigma_{p}$; m étant un coefficient adimensionnel pour lequel des valeurs comprises entre 0,75 et 0,85 ont été déterminées expérimentalement pour sept argiles de différentes

plasticités.
Les valeurs de $\frac{c_{u}^{n c}}{\sigma_{p}^{\prime}}$ et de $\frac{c_{y}^{n c i}}{\sigma_{p i}^{\prime}}$ ne sont pas égales mais restent du même ordre:

- selon Ladd (1965), leur écart relatif est au maximum de $\pm 15 \%$;

- les essais réalisés par Mitachi et Kitago (1976) sur trois argiles remaniées indiquent que $\frac{c_{u}^{n c}}{\sigma_{p}^{*}}$ est inférieur de 5 à $12 \%$ à $\frac{c_{\mu y}^{\text {nd }}}{\sigma_{p j}^{\prime}}$.

Par ailleurs, Mayne (1985), à partir de l'étude statistique de nombreux résultats d'essais de cisaillement effectués sur des argiles surconsolidées, propose la relation:

$$
\left(\frac{c_{u}}{\sigma_{v}^{\prime}}\right)_{K_{0}}=0,87\left(\frac{c_{u}}{\sigma_{v}^{\prime}}\right)_{t}
$$

$\left(\frac{c_{u}}{\sigma_{v}^{\prime}}\right)_{K_{0}}$ étant la cohésion réduite d'une argile surconsolidée, consolidée à déformation horizontale nulle sous la contrainte verticale $\sigma_{v}^{\prime}$ et $\left(\frac{c_{v}}{\sigma_{v}^{\prime}}\right)_{t}$ la cohésion réduite de la même argile consolidée sous une contrainte isotrope égale à $\sigma_{v^{*}}$ forme

Les relations (1) et (2) peuvent se mettre sous la

$$
\frac{c_{u}}{c_{u}^{n c}}=\left(\frac{\sigma_{v}}{\sigma_{p}^{\prime}}\right)^{1-m}
$$

$$
\text { et } \quad \frac{c_{u}}{c_{u j}^{n i}}=\left(\frac{\sigma_{c}^{\sigma}}{\sigma_{p i}^{\prime}}\right)^{1-m}
$$

Si l'on introduit les cohésions $c_{v}^{\sigma_{v}}$ et $c_{u}^{\sigma}$ cr correspondant respectivement à la pression de consolidation verticale $\sigma_{y T}^{\prime}$ et à la contrainte de consolidation isotrope $\sigma_{c r}^{\prime}$ prises comme référence, $\left(1^{\prime}\right)$ et $\left(2^{\prime}\right)$ deviennent : 


$$
\frac{c_{u}}{c_{u}^{\sigma_{w r}}}=\left(\frac{\sigma_{v}^{\prime}}{\sigma_{v r}^{\prime}}\right)^{1-m}
$$

et

$$
\text { et } \frac{c_{u}}{c_{u}^{\sigma_{c r}^{\prime}}}=\left(\frac{\sigma_{c}^{\prime}}{\sigma_{c r}^{\prime}}\right)^{1-m}
$$

L'analyse des résultats des essais au cône montre que les relations précédentes décrivent bien le comportement non drainé de l'argile des Flandres. Si l'on reporte sur un diagramme bilogarithmique les points de coordonnées $\frac{\sigma_{v}^{\prime}}{\sigma_{v r}}$ et $\frac{c_{u}}{c_{u}^{\alpha_{u}}}$ (Fig. 10), on constate, en effet, qu'ils se groupent autour d'une droite moyenne de pente $1-\mathrm{m}=0,26$, c'est-à-dire telle que $m=0,74$, valeur très proche de celles publiées antérieurement.

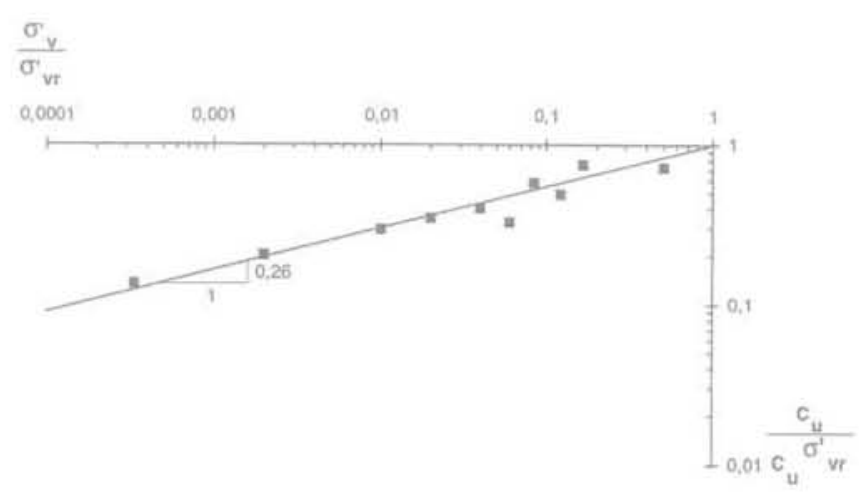

Fig. 10 Détermination de la relation entre la cohésion non drainée et la pression de consolidation à partir cles essais au pénétromètre de laboratoire.

Determination of the relationship between undrained shear strength and consoliciation pressure using fall cone test results.

\section{Cohésion non drainée de l'argile en place}

\section{3 .10 \\ Données expérimentales}

L'étude de la cohésion non drainée de l'argile en place s'appuie sur les résultats de l'ensemble des essais en place (à l'exception des essais au pressiomètre Ménard effectués à Dunkerque) et des essais de laboratoire non drainés (essais triaxiaux et de cisaillement direct de type UU) qui ont été réalisés lors des campagnes de reconnaissance du QPO et du QEP. Elle tient également compte des résultats des deux essais triaxiaux UU effectués au LCPC dans le cadre de l'étude du comportement de l'argile.

Les valeurs de la cohésion non drainée déduites des essais en place ont été obtenues comme suit:

- dans le cas des essais au pressiomètre autoforeur, à partir de la courbe de cisaillement déterminée par dérivation de la courbe d'expansion (Baguelin et al., 1972) ;

- dans le cas des essais au pressiomètre Ménard, au piézocône et au dilatomètre, par application de corrélations empiriques reliant un paramètre attaché à l'essai à la cohésion non drainée déterminée au moyen d'essais d'autres types (scissomètre, essais de laboratoire).

Les corrélations utilisées dans cette étude sont les suivantes :

- pressiomètre Ménard: $\quad c_{u}=\frac{p_{1}-p_{0}}{5,5}$

$p$, étant la pression limite.

$p_{0}$ la contrainte horizontale totale au niveau de l'essai, calculée en tenant compte d'une valeur de $\mathrm{K}$ égale à l'unité ;

- piézocône: $c_{u}=\frac{p_{t}-\sigma_{v a}}{N_{x}}$

$p$, étant la résistance de pointe corrigée, qui augmente quasi linéairement avec la profondeur et varie de $2,5 \mathrm{MPa}$ et $4,2 \mathrm{MPa}$ sur les dix premiers mètres de la couche d'argile,

$N_{K}$ le facteur de cône auquel on a affecté successivement les valeurs 10 et 12 ;

- dilatomètre Marchetti :

$c_{u}=0,22 \sigma_{s o}^{\prime}\left(0,5 K_{D}\right)^{1,25} \quad$ (Marchetti, 1980)

$\mathrm{K}_{\mathrm{D}}$ étant l'indice de contrainte horizontale.

L'ensemble des valeurs de $c_{\text {int }}$ ont été reportées sur les figures 11 (essais en place) et 12 (essais de laboratoire) en fonction de la profondeur d'essai (ou de prélèvement des éprouvettes essayées en laboratoire) comptée à partir du toit de l'argile. Les résultats obtenus sont discutés ci-après.

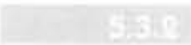

\section{Essais en place}

Les essais au scissomètre et au pressiomètre autoforeur sont les seul essais en place « autonomes », c'està-dire qui permettent d'atteindre des valeurs de la cohèsion non drainée à partir d'une interprétation rationnelle des mesures effectuées au cours de leur réalisation et indépendante de corrélations empiriques.

Les valeurs a priori les moins discutables de la cohésion non drainée de l'argile des Flandres sont donc celles tirées de ces deux essais. Ceux-ci conduisent à des valeurs élevées mais plausibles de $\mathrm{c}_{\mathrm{u}}$. Elles sont, en effet, largement inférieures aux valeurs de la cohésion de l'argile non fissurée dont un ordre de grandeur peut être estimé à partir de la relation :

$$
\frac{c_{u}}{\sigma_{v o}^{\prime}}=\frac{c_{u}^{n c}}{\sigma_{p}^{\prime}}\left(\frac{\sigma_{p}^{\prime}}{\sigma_{v e}^{\prime}}\right)^{m}
$$

précédemment citée (cf. \$ 5.2) et de la relation de Skempton (1957, citée par Bishop et Henkel, 1962) :

$$
\frac{c_{u c}^{n c}}{\sigma_{p}}=0,11+0,0037 I_{p}
$$




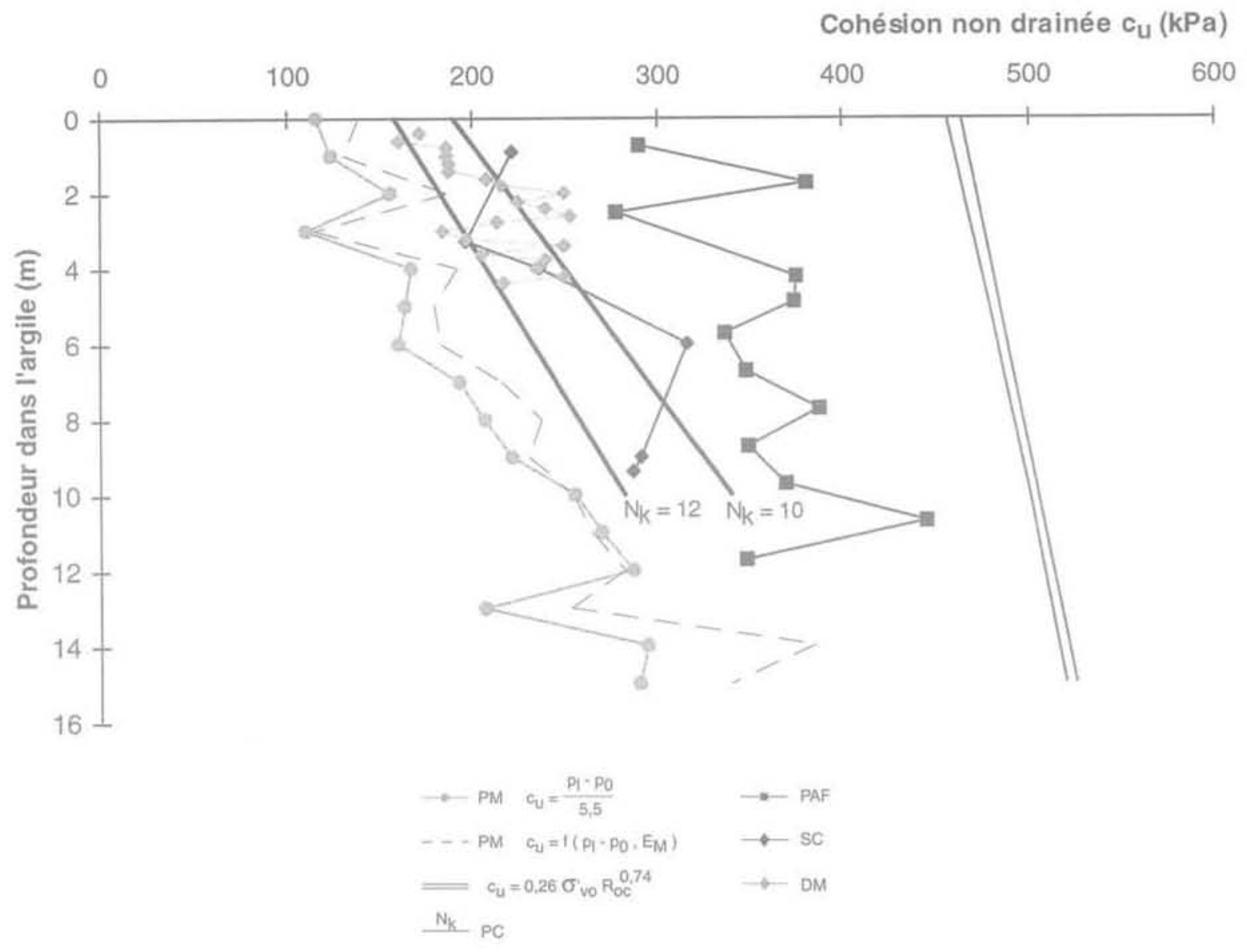

FG. 11 Variation avec la profondeur de la cohésion non drainée déterminée à partir des essais en place : essais au scissomètre de chantier ( $\mathrm{SC}$ ), au pressiomètre autoforeur (PAF), au pressiomètre Ménard (PM), au dilatomètre Marchetti (DM) et au piézocône (PC). Tous ces essais ont été réalisés à Calais à l'exception des essais au pressiomètre autoforeur qui ont été effectués à Dunkerque.

Undrained shear strength derived from in situ tests - field vane tests (SC), selfboring pressuremeter tests (PAF), Ménard pressuremeter tests (PM), Marchetti dilatometer tests (DM) and piezocone tests (PC) - versus depth. All these tests were performed at Calais save the selfboring pressuremeter tests which were carried out at Dunkirk.

La combinaison de ces deux relations et la prise en compte de l'indice de plasticité $I_{p} \approx 41 \%$ de l'argile des Flandres et de la valeur $\mathrm{m}=0,74$ déterminée précédemment (cf. § 5.2) conduit à :

$$
\frac{c_{u}}{\sigma_{v o}^{\prime}}=0,26\left(\frac{\sigma_{p}}{\sigma_{v o}^{\prime}}\right)^{0,74}
$$

La pression de préconsolidation de l'argile des Flandres étant de l'ordre de $3100 \mathrm{kPa}$ au niveau du toit de la couche, cette dernière expression indique que la cohésion de l'argile non fissurée serait d'environ $460 \mathrm{kPa}$ à la partie supérieure de la couche pour atteindre $500 \mathrm{kPa}$ à $10 \mathrm{~m}$ au-dessous de celle-ci, c'està-dire des valeurs supérieures de $50 \mathrm{kPa}$ à près de $200 \mathrm{kPa}$ (en moyenne d'environ $100 \mathrm{kPa}$ ) à celles mesurées au pressiomètre autoforeur, elles-mêmes supérieures à celles mesurées au scissomètre.

La position relative des profils de résistance obtenus au pressiomètre autoforeur, d'une part, et au scissomètre, d'autre part, est la même que celle obtenue par Amar et al. (1975) dans le cas de la plupart des sols argileux de consistance faible à moyenne testés à l'aide de ces deux équipements. Pour neuf des onze sols étu- diés par ces auteurs, les valeurs de $c_{\text { déterminées au }}$ pressiomètre autoforeur étaient, en effet, supérieures à celles déterminées au scissomètre. Le rapport des résistances variait de 1,20 à 1,80 pour ces neuf matériaux et de 1,2 à 1,56 pour huit d'entre eux. Ces résultats sont donc cohérents avec les mesures effectuées sur l'argile des Flandres puisque le rapport des valeur's moyennes de $c_{\text {y }}$ obtenues au pressiomètre autoforeur et au scissometre est d'environ 1,5 sur les cinq premiers mètres de la couche et de 1,2 sur les cing mètres suivants.

L'essai au pressiomètre autoforeur, qui sollicite un matériau très peu remanié par l'introduction de la sonde, donne des valeurs de la résistance non drainée proches de celles du sol intact. Comme on l'a vu précédemment, ces valeurs sont à peu près toujours supérieures à celles obtenues à partir de l'essai au scissomètre qui remanie davantage le sol et met vraisemblablement en jeu un mécanisme de rupture progressive (Amar et al., 1975). On peut donc conclure que la cohésion non drainée de l'argile des Flandres est caractérisée par des valeurs intermédiaires entre celles obtenues au pressiomètre autoforeur et au scissomètre et, qu'en tout état de cause, elle est au moins égale à $200 \mathrm{kPa}$ sur les cinq premiers mètres de la couche et à $300 \mathrm{kPa}$ à une profondeur supérieure. 


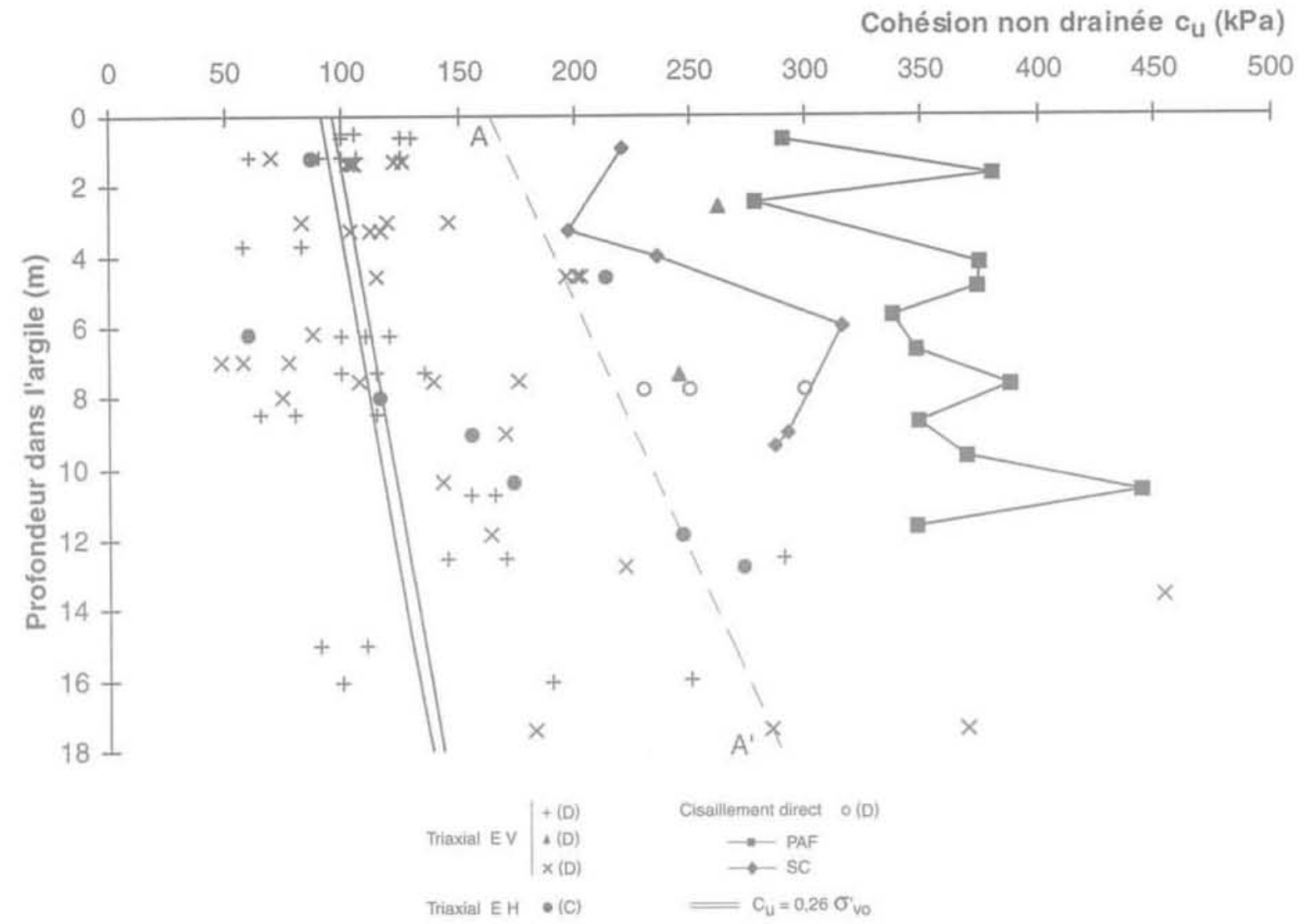

Variation avec la profondeur de la cohésion non drainée déterminée à partir des essais triaxiaux UU effectués sur éprouvettes verticales (EV) et horizontales (EH) et à partir d'essais de cisaillement direct UU. Les résultats des essais au scissomètre de chantier (SC) et au pressiomètre autoforeur (PAF) ont également été représentés aux fins de comparaison. La lettre (C) ou (D) indique le site auquel se rapportent les essais (Calais ou Dunkerque).

Undrained shear strength derived from UU triaxial tests performed on vertical (EV) and horizontal (EH) specimens as well as from UU direct shear versus depth. Field vane (SC) and selfboring pressuremeter (PAF) test results have also been plotted for the sake of comparison. The letter (C) or (D) stands for the site which the tests are related to (Calais or Dunkirk),

On remarque, par ailleurs, que les deux profils de résistance obtenus à partir des essais au piézocône pour les valeurs $\mathrm{N}_{\mathrm{K}}=10$ et $\mathrm{N}_{\mathrm{K}}=12$ encadrent une grande partie des points représentatifs de la cohésion tirée des essais au scissomètre et au dilatomètre. Il en résulte que, dans le cas particulier de l'argile des Flandres, l'exploitation des essais au dilatomètre à partir de la corrélation de Marchetti, $c_{u}=0,22 \sigma_{s o}\left(0,5 K_{p}\right)^{1,25}$, et des essais au piézocône en tenant compte d'un facteur de cône compris entre 10 et 12, permet de retrouver un ordre de grandeur de la cohésion non drainée mesurée au scissomètre.

On constate également que le profil de résistance déterminé à partir des essais au pressiomètre Ménard

en appliquant la corrélation $\quad c_{u}=\frac{p_{l}-p_{0}}{5,5} \quad$ conduit à

des valeurs de $c_{y}$ sensiblement inférieures à celles tirées des essais au scissomètre et au piézocône, mais qui restent malgré tout du même ordre. La concordance est d'ailleurs sensiblement améliorée, tout au moins en haut de couche, lorsqu'on calcule $c_{u}$ au moyen de la formule de Ménard: $p_{1}-p_{0}=\left[1+\ln \frac{E_{M}}{2 c_{u}(1+v)}\right]$ (citée par Combarieu , 1995)

en affectant au coefficient de Poisson $v$ la valeur conventionnelle 0,33 .

En revanche, les corrélations établies pour les argiles raides (Amar et Jézéquel, 1972; Cassan, 1988) conduisent à des valeurs de $c_{u}$ beaucoup plus faibles; qui sont sans rapport avec celles tirées des autres essais en place. Ceci est vraisemblablement dû au fait que ces corrélations ont été obtenues par référence à des essais de laboratoire effectués sur des éprouvettes plus ou moins remaniées. Une observation analogue peut être faite à propos du coefficient $\mathrm{N}_{K}$ qui intervient dans l'interprétation des essais de pénétration statique, pour lesquels des valeurs très élevées ont été proposées dans le cas de certaines argiles.

\subsection{3}

\section{Essais de laboratoire}

Les 82 valeurs de $\mathrm{c}_{4}$ déterminées à partir des essais de laboratoire, c'est-a-dire pour l'essentiel à partir d'essais triaxiaux, se situent dans la plage $48 \mathrm{kPa}$ - 
$633 \mathrm{kPa}$, une seule d'entre elles étant supérieure à $454 \mathrm{kPa}$ et 18 étant comprises dans l'intervalle $196 \mathrm{kPa}$ $454 \mathrm{kPa}$. Cette dispersion considérable est liée au remaniement et à la fissuration de nombreuses éprouvettes.

L'inclinaison des plans de rupture n'a pas été relevée dans le cas des essais triaxiaux mais il est possible, dans une certaine mesure, de différencier les éprouvettes initialement fissurées de celles qui ne l'étaient pas, ceci en étudiant les variations de $\mathrm{c}_{u}$ en fonction de la teneur en eau W. A l'hétérogénéité du sol près (qui inclut les différences de degré de remaniement) les variations de la cohésion non drainée du sol non fissuré sont, en effet, régies par les variations de la teneur en eau w.

La cohésion non drainée $c_{u}$ d'une argile surconsolidée, consolidée sous la contrainte isotrope $\sigma{ }^{\prime}$, satisfait à la relation $\left(2^{\prime \prime}\right)$

$$
\frac{c_{y}}{c_{u t}^{\sigma_{\sigma o}}}=\left(\frac{\sigma_{c}^{\prime}}{\sigma_{c r}^{\prime}}\right)^{1-m}
$$

dans laquelle $c_{u}^{\sigma}$ cr désigne la cohésion non drainée de la même argile consolidée sous la contrainte isotrope $\sigma_{c}^{\prime}$, prise comme référence,

En outre, les valeurs e et $e$, de l'indice des vides qui définissent l'état de l'argile sous les contraintes $\sigma$, et $\sigma_{\text {cr }}^{\prime}$ sont liées à ces dernières et à l'indice de gonflement $\mathrm{C}_{\mathrm{s}}$ sous contrainte isotrope par la relation :

$$
C_{s}=-\frac{e-e_{r}}{\lg \frac{\sigma_{c}^{\prime}}{\sigma_{c r}^{\prime}}}
$$

En introduisant les valeurs w et w, correspondantes de la teneur en eau, il vient:

$$
C_{s}=-\frac{\gamma_{s}}{\gamma_{w}} \frac{W-w_{r}}{\lg \frac{\sigma_{c}^{\prime}}{\sigma_{c r}^{\prime}}}
$$

On a par ailleurs :

$$
\lg \frac{c_{u}}{c_{u}^{\sigma_{c}}}=(1-m) \lg \frac{\sigma_{c}^{\prime}}{\sigma_{\sigma}^{\prime}}
$$

d'où:

$$
\lg \frac{c_{y}}{c_{u}^{\sigma_{\sigma}}}=\frac{\gamma_{s}}{\gamma_{w}} \frac{m-1}{C_{s}}\left(w-w_{r}\right)
$$

ou encore :

$$
c_{\mu}=c_{u}^{\sigma_{\sigma r}^{\prime}} \times 10^{\frac{\gamma_{z}}{\gamma_{u}}} \frac{m-1}{c_{s}}\left(w-w_{r}\right)
$$

Les valeurs de l'indice de gonflement qui caractérisent les variations de volume d'une argile déchargée, sous contrainte isotrope, d'une part, et à déformation latérale nulle, d'autre part, étant pratiquement égales (Mitachi et Kitago, 1976), on a affecté à ce paramètre la valeur $C_{\mathrm{s}}=0,1$ tirée des essais œdométriques. Comme on a par ailleurs $\gamma_{\epsilon}=27,5 \mathrm{kN} / \mathrm{m}^{3}$ et $\mathrm{m}=0,74$, l'expression précédente devient:

$$
c_{u}=c_{u}^{\sigma o r} \times 10^{-7.75 / w-w r i}
$$

Le diagramme de la figure 13 , sur lequel ont été reportées les valeurs de $c_{u}$ en fonction de $w$ et oú ont été tracées trois courbes caractéristiques de variation de $c_{u^{\prime}}$ montre :

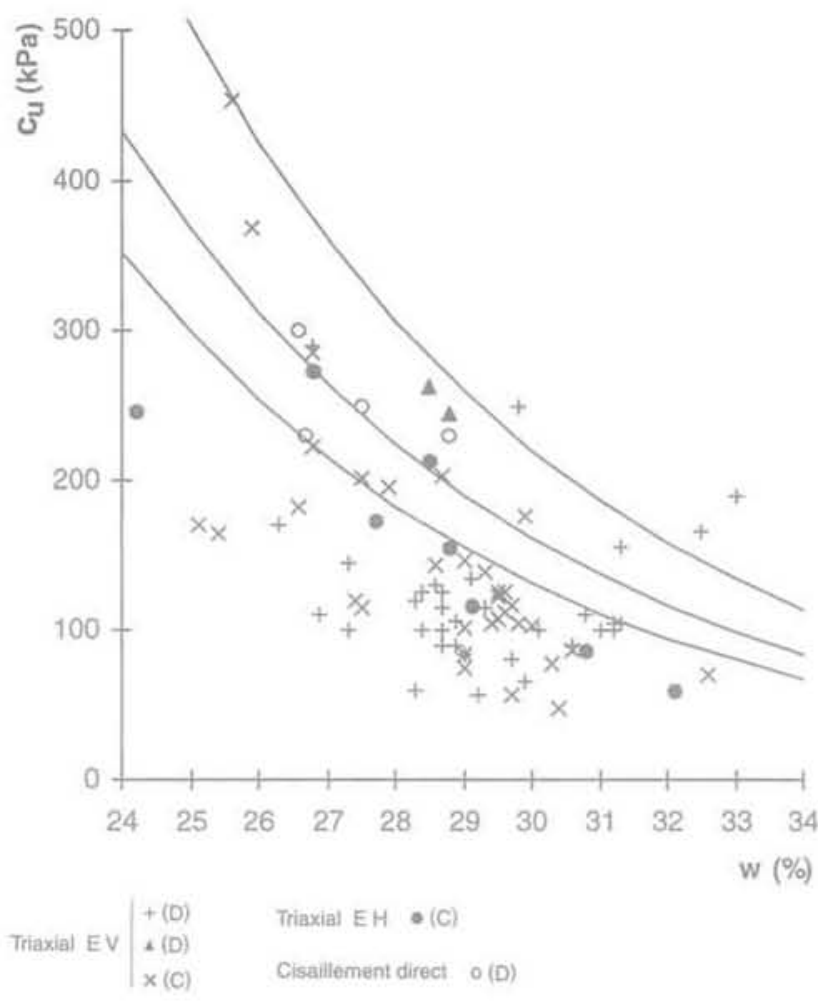

FiG. 13 Variation de la cohésion non drainée déterminée à partir des essais UU de laboratoire en fonction de la teneur en eat des éprouvettes.

Relationship between the undrained shear strength derived from the laboratory UU tests and the water content of the specimens.

- que tous les points correspondant à des valeurs de c supérieures à $196 \mathrm{kPa}$ sont compris dans le fuseau délimité par les deux courbes extrêmes, à l'exception de trois d'entre eux (dont le point de coordonnées $c_{i 4}=633 \mathrm{kPa}$ et $\mathrm{W}=25,1 \%$ non représenté)

- que douze des points précédents sont compris dans le fuseau supérieur.

Ceci montre que, dans le cas de la plupart des éprouvettes ayant une cohésion supérieure à $196 \mathrm{kPa}$, la cohésion de l'argile est liée principalement à sa teneur en eau, ce qui implique que le sol n'était pas fissuré initialement. Par suite, les essais correspondants donnent des valeurs de la cohésion non drainée de la matrice argileuse plus ou moins affectées par le remaniement. Si on compare leurs résultats avec ceux des essais en place, ils semblent fournir des valeurs acceptables de c (particulièrement ceux dont les points représentatifs se situent à droite de la ligne $\mathrm{AA}^{\prime}$ 'représentée sur la figure 12). On notera que les essais UU effectués au LCPC (représentés par des triangles) sur des éprouvettes de relativement bonne qualité appartiennent à ce groupe d'essais.

En ce qui concerne le groupe d'éprouvettes sur lesquelles on a mesuré une cohésion inférieure à $196 \mathrm{kPa}$, il apparait que les plus résistantes ne sont pas fissurées mais remaniées, tandis que les très faibles valeurs de $c$ obtenues à partir des autres s'expliquent soit par la fissuration du sol, soit par un remaniement important ou encore par la combinaison des deux. Le caractère non 
représentatif des résultats des essais effectués sur les éprouvettes de ce groupe peut également ètre mis en évidence en comparant les valeurs mesurées de $c_{u}$ avec celles qui caractériseraient l'argile si elle était normalement consolidée sous les contraintes en place actuelles. La cohésion non drainée $\mathrm{c}_{u}^{n c}$ de l'argile normalement consolidée peut être évaluée par la relation de Skempton :

$$
\frac{c_{H}^{n c}}{\sigma_{v o}^{\prime}}=0,11+0,0037 I_{p .}
$$

soit, dans le cas de l'argile des Flandres, pour laquelle $I_{p} \approx 41 \%$ :

$$
c_{w}^{n x}=0,26 \sigma_{\text {vo }}^{\prime}
$$

On constate (Fig. 12) que, bien que le rapport de surconsolidation de l'argile varie approximativement de 9 à 6 sur les 20 mètres supérieurs de la couche (valeurs calculées pour $\sigma_{p}^{\prime}=3100 \mathrm{kPa}$ en toit de couche), les valeurs mesurées de $c_{u}$ sont de l'ordre de $\mathrm{C}_{\mathrm{u}}^{\mathrm{nc}}$, ce qui met en évidence leur caractère irréaliste.

L'analyse précédente montre qu'environ $20 \%$ des résultats des essais UU conduisent à des valeurs acceptables de la cohésion non drainée de l'argile (23\% si l'on tient compte de l'ensemble des essais de laboratoire, $17 \%$ si l'on ne considère que les essais triaxiaux), ceci en raison de la fissuration et du remaniement des éprouvettes. Elle fait donc clairement ressortir la nécessité, non seulement de réaliser les essais sur un matériau aussi peu remanié que possible, mais également de pouvoir contrôler la validité des résultats des essais. Pour ce faire, on doit pouvoir comparer la contrainte isotrope initiale effective $p^{\prime}$, à la contrainte moyenne effective en place $p^{\prime}$, la résistance mesurée pouvant être considérée comme représentative lorsqu'elles sont du méme ordre (Padfield et Mair, 1984). Ceci impose de mesurer systématiquement $p^{\prime}$, c'est-à-dire la contrainte isotrope de gonflement dans le cas des sols saturés. Une telle procédure augmente substantiellement la durée et la complexité de l'essai UU, mais est indispensable à une détermination fiable de la cohésion non drainée au moyen de ce type d’essai (Baldi et al., 1988).

\section{6}

\section{Résistance au cisaillement drainé}

L'étude de la résistance au cisaillement drainé s'appuie sur l'analyse en contraintes effectives des résultats des essais triaxiaux de tous types effectués au LCPC dans le cadre de l'étude du comportement de l'argile et sur les résultats des essais de cisaillement direct de type consolidé drainé réalisés au LRPC de Lille lors de l'étude du QEP.

\section{1}

\section{Essais triaxiaux}

On a tout d'abord tracé les enveloppes de rupture en compression et en extension dans le plan $\mathrm{s}^{\prime}=\frac{\sigma_{u}^{\prime}+\sigma_{f}^{\prime}}{2}$. $t=\frac{\sigma_{i}-\sigma_{r}}{2}$ à partir des points de coordonées s, et $t_{t}$ représentatifs de l'état des contraintes principales appliquées aux éprouvettes au moment de la rupture. Cette procédure, qui est la procédure usuelle d'interprétation des essais, n'est applicable en toute rigueur que dans le cas de sols homogènes qui ne comportent pas de fissures préexistantes, celles-ci constituant des surfaces préférentielles de rupture. L'inclinaison $\alpha$ du plan de rupture sur l'horizontale est alors égale à $\frac{\pi}{4}+\frac{\varphi^{\prime}}{2}$ dans le cas des essais en compression et à $\frac{\pi}{4}-\frac{\varphi^{\prime}}{2}$ dans le cas des essais
en extension.

L'inclinaison des plans de rupture observés étant souvent très différente de ces valeurs, on a également déterminé les enveloppes de rupture en reportant dans le plan $\left(\sigma^{\prime}, \tau\right)$ les points de coordonnées $\sigma_{\text {, et }} \tau$, qui désignent les composantes normale et tangentielle de la contrainte appliquée au plan de rupture au moment de la rupture et qui sont calculées à partir des expressions:

et

$$
\begin{gathered}
\sigma_{f}^{\prime}=s_{f}^{\prime}+t_{f} \cos 2 \alpha \\
\tau_{f}=t_{f} \sin 2 \alpha .
\end{gathered}
$$

\section{4.thif}

\section{Interprétation usuelle}

L'examen du diagramme de la figure 14 montre que la majeure partie des points représentatifs des essais en compression sont localisés dans un fuseau du plan $\left(s^{\prime}, t\right)$, la quasi-totalité des points représentatifs des essais en extension étant situés dans un fuseau pratiquement symétrique par rapport à l'axe des abscisses. Chacun de ces fuseaux est limité par deux droites parallèles définies par les paramètres suivants :

$c^{\prime}=0, \varphi^{\prime}=23,4^{\circ}$ et $c^{\prime}=109 \mathrm{kPa}, \varphi^{\prime}=23,4^{\circ}$ dans le cas des essais en compression :

$c^{\prime}=0, \varphi^{\prime}=23,4^{\circ}$ et $c^{\prime}=88 \mathrm{kPa}, \varphi^{\prime}=23,4^{\circ}$ dans le cas des essais en extension:

les valeurs moyennes des paramètres de résistance correspondants (obtenus par régression linéaire) étant: $c^{\prime}=36 \mathrm{kPa}$ et $\varphi^{\prime}=24,3^{\circ}$ en compression avec $\mathrm{r}=0,975$; $c^{\prime}=59 \mathrm{kPa}$ et $\varphi^{\prime}=21,7^{\circ}$ en extension avec $\mathrm{r}=0,924$; $r$ désignant le coefficient de corrélation de la régression.

\subsection{7}

\section{Interprétation tenant compte de l'inclinaison des plans de rupture}

La prise en compte de la résistance mobilisée le long des plans de rupture conduit à des résultats sensiblement différents (Fig. 15).

On constate, en effet, que la plupart des points représentatifs des essais en compression sont situés dans deux fuseaux bien distincts du diagramme $(\sigma, \tau)$, chacun d'eux étant délimité par deux droites définies par les paramètres suivants :

$c^{\prime}=0, \varphi^{\prime}=23,3^{\circ}$ et $c^{\prime}=92 \mathrm{kPa}, \varphi^{\prime}=23,3^{\circ}$ pour le fuseau I; $c^{\prime}=0, \varphi^{\prime}=11,4^{\circ}$ et $c^{\prime}=0, \varphi^{\prime}=16,3^{\circ}$ pour le fuseau II; les valeurs moyennes des paramètres de résistance associées aux deux groupes d'essais correspondants étant respectivement : 


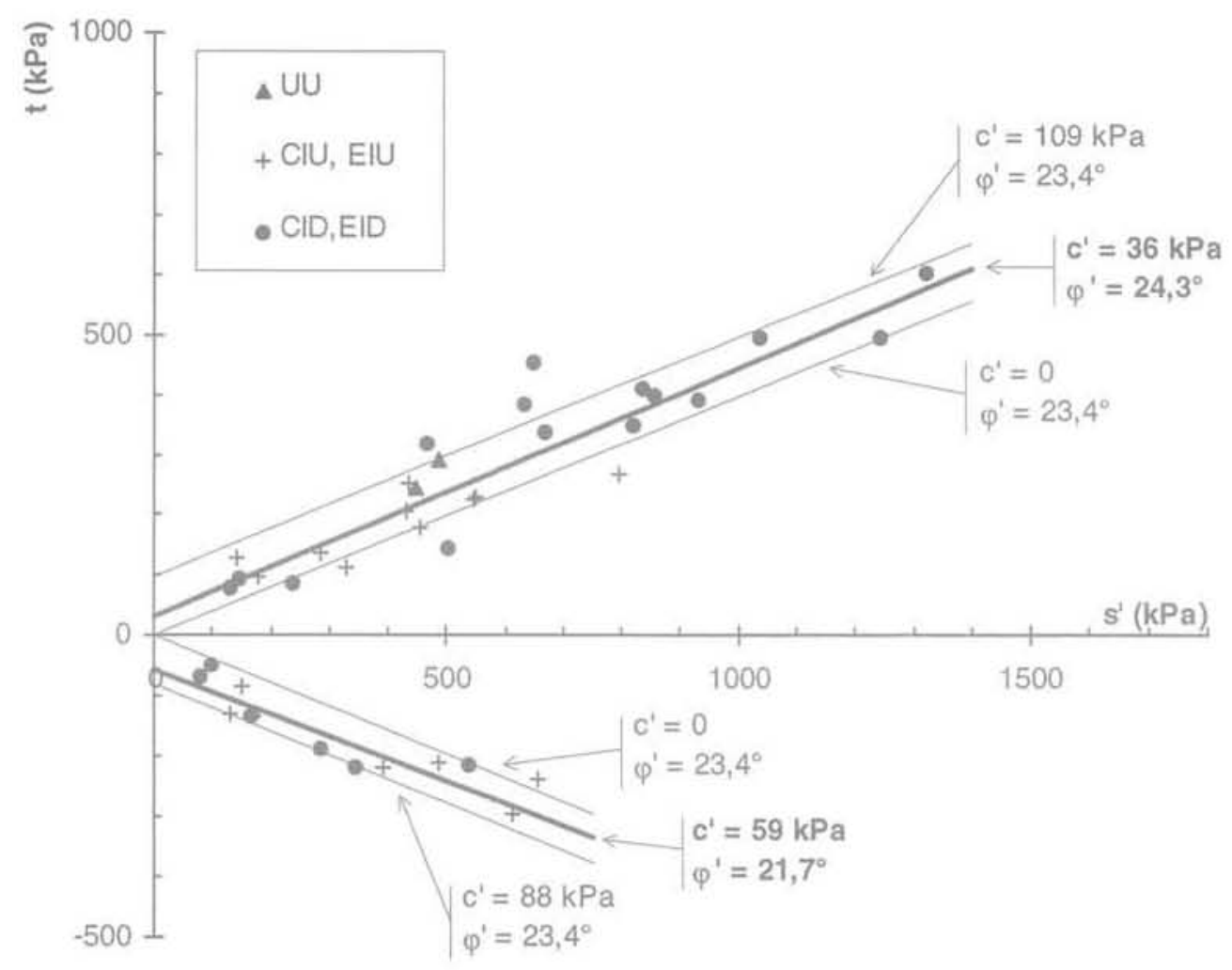

FIG.14 Enveloppes de rupture obtenues en supposant les éprouvettes parfaitement homogènes. Failure envelopes obtained on the assumption that the specimens were perfectly homogeneous.

$c^{\prime}=29 \mathrm{kPa}$ et $\varphi^{\prime}=23,7^{\circ}$ pour le groupe I avec $\mathrm{r}=0,974$; $c^{\prime}=0$ et $\varphi^{\prime}=14^{\circ}$ pour le groupe II avec $\mathrm{r}=0,94$.

On remarque que les paramètres déduits des essais du groupe I sont très voisins de ceux déterminés à partir des essais en compression interprétés par la méthode usuelle (cf. Fig. 14).

Il apparait en outre que les deux groupes d'essais I et II se différencient par l'inclinaison des plans de rupture des éprouvettes:

- les inclinaisons des plans de rupture mesurées sur les 14 éprouvettes du groupe I varient de $32^{\circ}$ à $62^{\circ}$. Toutefois à huit de ces éprouvettes, dont les points représentatifs se répartissent à peu près uniformément sur toute la surface du fuseau I, sont associées des valeurs de $\alpha$

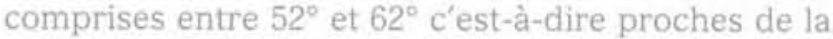

valeur $\frac{\pi}{4}-\frac{\varphi^{\prime}}{2}$ (égale à environ $56^{\circ}-57^{\circ}$ ) et encadrant

celle-ci. Il s'ensuit, qu'au moins dans le cas de ces 8 éprouvettes, la rupture s'est développée dans la matrice argileuse et que la résistance au cisaillement de cette dernière est caractérisée par les paramètres de résistance déterminés à partir des essais du groupe I, ou par des paramètres très voísins ;

- en revanche, les éprouvettes du groupe II se sont rompues suivant des plans faiblement inclinés sur l'horizontale ( $\alpha$ était compris entre $18^{\circ}$ et $26^{\circ}$ pour 6 des 7 éprouvettes du groupe), le long desquels la résistance mobilisée est très inférieure à la résistance de la matrice argileuse. Ceci indique que la rupture s'est produite suivant des plans de glissement préexistants le long desquels la résistance disponible est probablement égale à la résistance résiduelle.
Dans cette hypothèse, les paramètres de résistance résiduelle seraient:

$$
c_{r}=0 \varphi_{r}^{\prime} \approx 14^{\circ}
$$

Les essais en extension peuvent être classés en trois groupes définis par référence aux valeurs de l'inclinaison $\alpha$ des plans de rupture, celles-ci étant réparties dans les intervalles $8^{\circ}-11^{\circ}$ ( 2 essais), $16^{\circ}-21^{\circ}$ ( 5 essais) et $28^{\circ}-34^{\circ}$ ( 4 essais). Ces groupes ne comportant qu'un petit nombre d'essais dont les résultats sont, en outre, relativement dispersés (Fig. 15), il n'est pas possible de différencier les paramètres de résistance caractérisant chacun d'eux.

On se bornera donc à constater que les points représentatifs des essais en extension, à l'exception d'un seul, sont contenus dans un fuseau du plan $\left(\sigma^{\prime}, \tau\right)$ limité par deux droites parallèles définies par les paramètres suivants :

$$
c^{\prime}=0, \varphi^{\prime}=21,2^{\circ} \text { et } c^{\prime}=90 \mathrm{kPa}, \varphi^{\prime}=21,2^{\circ},
$$

dont les valeurs sont assez proches de celles qui définissent le fuseau des points représentatifs des essais en extension interprétés par la méthode usuelle (cf. Fig. 14).

\section{2}

\section{Essais de cisaillement direct}

Les essais de cisaillement direct donnent des résultats très comparables à ceux des essais en compression interprétés par la méthode usuelle et à ceux des résultats des essais en compression du groupe I interprétés en tenant compte de l'inclinaison des plans de rupture. 


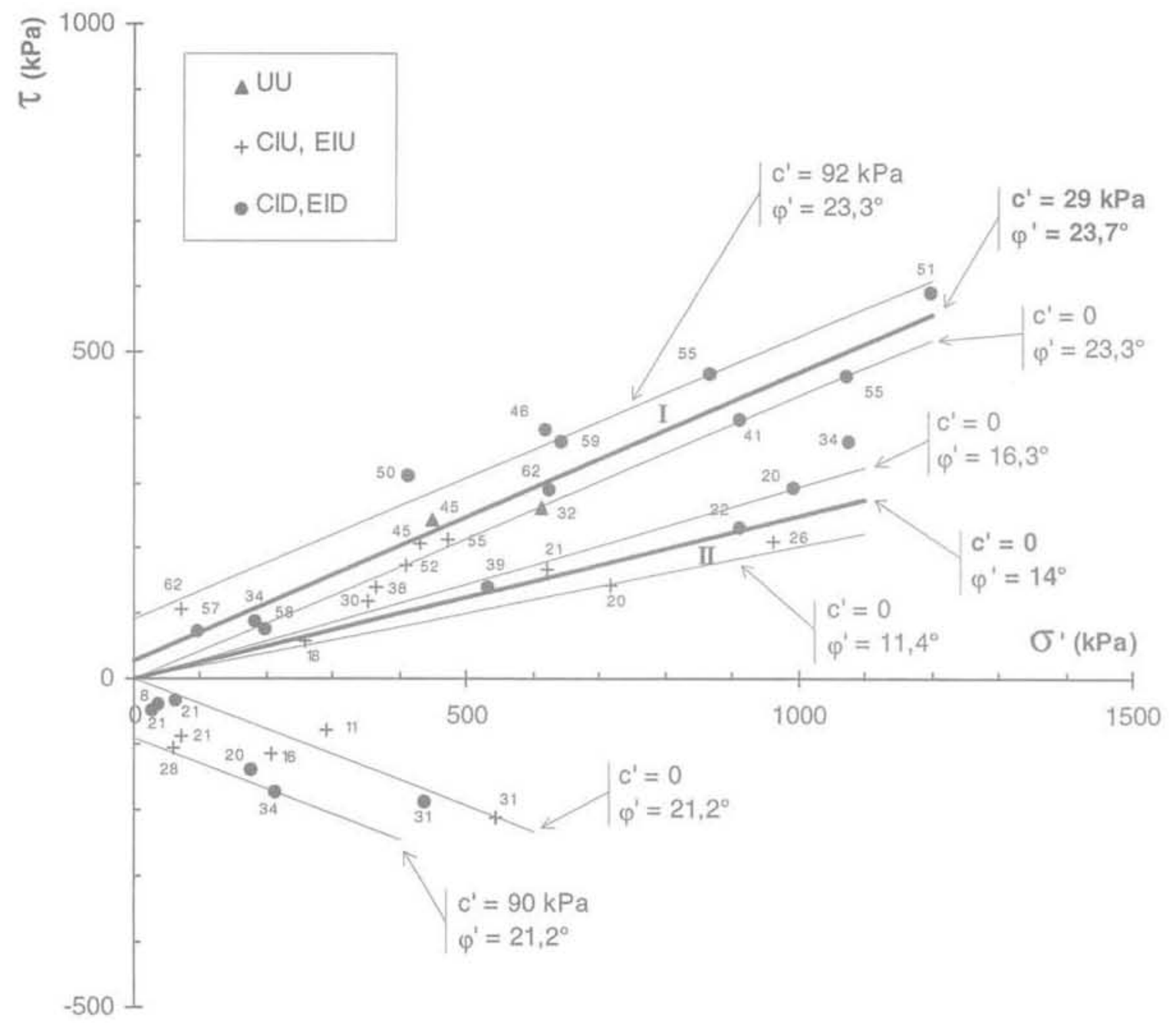

FIG. 15 Enveloppes de rupture obtenues en tenant compte de l'inclinaison réelle $\alpha$ des plans de rupture. Les nombres accolés aux points du diagramme sont les valeurs de $\alpha$ exprimées en degrés.

Failure envelopes taking into account the actual inclination $\alpha$ of the failure planes. The numbers next to the points of the diagram are the values of $\alpha$ expressed in degrees.

En effet, les droites qui délimitent le fuseau contenant les points représentatifs des essais de cisaillement dans le plan $\left(\sigma^{\prime}, \tau\right)$, (Fig. 16) sont définies respectivement par les paramètres $\mathrm{c}^{\prime}=52 \mathrm{kPa}, \varphi^{\prime}=23^{\circ}$ et $\mathrm{c}^{\prime}=$ $96 \mathrm{kPa}, \varphi^{\prime}=23^{\circ}$ et l'enveloppe de rupture moyenne est caractérisée par $c^{\prime}=52 \mathrm{kPa}, \varphi^{\prime}=23^{\circ}$ avec $r=0,839$.

Les éprouvettes soumises à l'essai de cisaillement direct étant cisaillées dans des plans horizontaux a priori non affectés par la fissuration de l'argile, la résistance mesurée est celle de la matrice argileuse ce qui confirme les conclusions de l'analyse des résultats des essais en compression du groupe I (cf $\$ 6.1 .2$ ).

\section{3}

\section{Paramètres de résistance}

Il ressort de l'analyse précédente que le mode de sollicitation des éprouvettes (compression ou extension) semble avoir peu d'influence sur la résistance au cisaillement drainé de la matrice argileuse. Celle-ci est caractérisée par un angle de frottement interne d'environ $23^{\circ}$ et par une cohésion qu'il est difficile d'évaluer avec précision, mais pour laquelle on pourra retenir la valeur $c^{\prime}=$ $30 \mathrm{kPa}$ (voisine de la plus petite valeur moyenne déterminée précédemment). On notera, toutefois, que la résis-

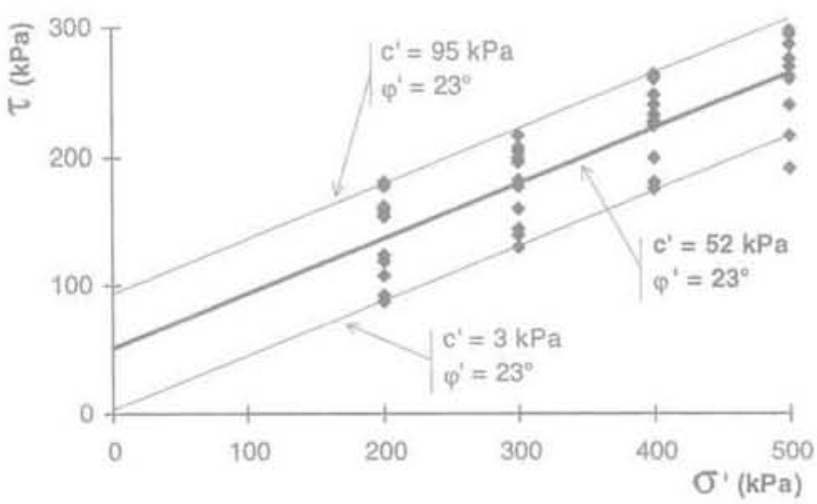

FG. 16 Enveloppe de rupture déterminée à partir des essais de cisaillement direct. Failure envelope derived from direct shear tests.

tance de la matrice argileuse est supérieure à la résistance globale du massif fissuré et que, de ce fait, la cohésion disponible en place peut être notablement inférieure à la valeur mentionnée précédemment. De plus, les paramètres de résistance mobilisés le long d'une surface de glissement préexistante, qui se confondent vraisemblablement avec les paramètres de cisaillement résiduel, ont pour valeurs moyennes $c^{\prime}=0$ et $\varphi^{\prime} \approx 14^{\circ}$. 


\section{Paramètres de déformabilité}

\section{1}

\section{Détermination à partir des essais triaxiaux}

Les principales informations concernant la déformabilité de l'argile ont été fournies par les essais triaxiaux. Ceux-ci ont en effet permis de déterminer : - les modules de déformation drainés et non drainés, en compression et extension, à partir des courbes contraintes-déformations obtenues au cours de la phase de cisaillement des essais CID et EID, d'une part, et des essais UU, CIU et EIU, d'autre part ;

- le coefficient de Poisson, à partir des courbes de variation de la déformation volumique $\varepsilon$ en fonction de la déformation axiale $\varepsilon_{a}$ obtenues au cours de la phase de cisaillement des essais CID et EID.

\section{Tatit \\ Résultats}

Les valeurs des modules $E_{0}$ (module tangent à l'origine), $\mathrm{E}_{\mathrm{r}}$ (module sécant à $\varepsilon \%$ de déformation axiale) et $E_{50}$ (module sécant à $50 \%$ du déviateur à la rupture), soit les valeurs:

- $E_{40,25}, E_{40.5,}, E_{41}$ et $E_{450}$ des modules non drainés ;

- $E_{0}^{\prime} E_{0.5}^{\prime}, E^{\prime}$, et $E_{50}^{\prime}$ des modules drainés ;

sont présentées dans les tableaux III et IV pour les essais en compression et en extension.

Les valeurs $E_{i 0}$ des modules non drainés tangents à l'origine n'ont pas été reportées dans le tableau III, nombre d'entre elles n'ayant pu être déterminées avec une fiabilité suffisante, en raison de difficultés d'appréciation de l'origine des courbes contraintes-déformations correspondantes.

On notera que les valeurs des modules déterminées à partir des essais triaxiaux sont entachées d'une erreur par défaut qui peut être importante. Cette erreur est liée, d'une part, à la qualité assez médiocre des éprouvettes d'essai, d'autre part, au fait que, comme dans tous les essais classiques, la déformation axiale de l'éprouvette est assimilée au déplacement relatif du piston et de la cellule triaxiale alors qu'elle est notablement plus faible, principalement dans le domaine des petites déformations (Jardine et al., 1984).

Les valeurs $v$ ' du coefficient de Poisson du squelette solide ont été déterminées à partir des valeurs de la pente à l'origine des courbes $(\varepsilon, \varepsilon)$ relatives à la phase de cisaillement des essais draînés, au moyen de l'expression :

$$
v^{\prime}=\frac{1}{2}\left[1-\left(\frac{d \varepsilon_{v}}{d \varepsilon_{a}}\right)_{\varepsilon_{a=0}}\right]
$$

Les valeurs obtenues sont relativement dispersées. Elles sont comprises entre 0,08 et 0,21 (moyenne 0,16 ) dans le cas des essais CID et entre 0,10 et 0,24, dans le cas des essais EID (moyenne 0,18), la moyenne générale s'établissant à 0,17 .

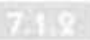

\section{Variation des modules de déformation avec la pression de consolidation}

Les valeurs des rapports $\frac{E}{\sigma_{t}^{*}}$ des différents mo-

dules définis précédemment à la pression de consolidation correspondante ont été reportées en fonction du paramètre $\frac{\sigma_{c o}}{\sigma_{c}^{\prime}}$ (égal à un facteur près au rapport de surconsolidation) dans un système de coordonnées bilogarithmique, $\sigma$ étant une contrainte de référence à laquelle on a attaché la valeur $1 \mathrm{MPa}$. Les diagrammes ainsi obtenus sont semblables au diagramme de la figure 17 relatif aux modules drainés initiaux tangents. L'examen de ces diagrammes montre que les points représentatifs des modules en extension sont très dispersés, alors que, à l'exception d'un ou deux, les points relatifs aux modules en compression se distribuent autour d'une droite moyenne d'équation :

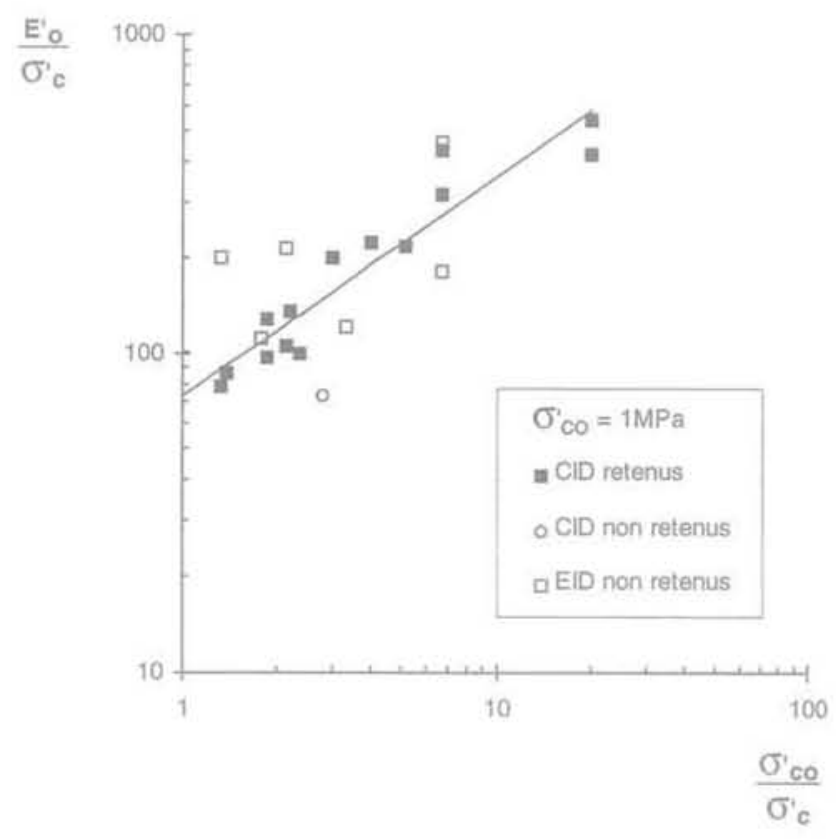

FiG. 17 Détermination de la relation empirique entre le module drainé initial tangent $E_{\text {. }}$ en compression et la contrainte isotrope de consolidation $\sigma$. Cette relation ne tient pas compte des modules en extension. Determination of the relationship between drained initial tangent modulus E' derived from compression tests and isotropic consolidation stress $\sigma$. This relationship does not take into account the moduli derived from extension tests.

$$
\lg \left(\frac{E}{\sigma_{c}^{\prime}}\right)=a+b \lg \left(\frac{\sigma_{c o}}{\sigma_{c}}\right) \quad \text { avec } a=\lg \left(\frac{E^{\sigma_{c o}}}{\sigma_{c o}^{\prime}}\right)
$$

$E^{\sigma_{c o}^{\prime}}$ étant la valeur de $E$ correspondant à $\sigma_{c}^{\prime}=\sigma_{c 0^{*}}$ La loi de variation de $E$ peut donc se mettre sous la forme:

$$
\frac{E}{\sigma_{c}^{\prime}}=\frac{E^{\sigma_{c 0}}}{\sigma_{c o}^{\prime}}\left(\frac{\sigma_{c o}^{\prime}}{\sigma_{c}^{\prime}}\right)^{b}
$$


ou encore :

$$
E=E^{\sigma_{c i t}}\left(\frac{\sigma_{c}^{\prime}}{\sigma_{c o}^{\prime}}\right)^{\beta} \operatorname{avec} \beta=1-b
$$

soit, compte tenu de $\sigma_{c o}=1 \mathrm{MPa}$ :

$$
E=E^{1}\left(\sigma^{\prime}\right)^{\beta}
$$

$E, E^{\prime}$ et $\sigma_{c}^{\prime}$ étant exprimés en $\mathrm{MPa}$ :

Les valeurs de $E^{\prime}$ et de $\beta$ obtenues par régression linéaire pour les différents modules considérés sont reportées dans le tableau VII ainsi que les valeurs de $E$ à la profondeur $\mathrm{z}^{*}=6 \mathrm{~m}$ dans l'argile $\left(\sigma^{\prime}=\sigma^{\prime}=\right.$ $0,445 \mathrm{MPa}$ ), c'est-à-dire à mi-hauteur de la zone reconnue au pressiomètre autoforeur.

\section{Th 3}

\section{Anisotropie de déformabilité}

L'anisotropie de déformabilité de l'argile est mise en évidence par la comparaison des valeurs $\varepsilon_{v c}$ et $\varepsilon_{n}$ des déformations volumique et axiale mesurées pendant la phase de consolidation isotrope. Pour la plupart des essais, les valeurs du rapport $\frac{\varepsilon_{\mathrm{vc}}}{\varepsilon_{\mathrm{ac}}}$ s'écartent, en effet, notablement de la valeur 3 qui correspond à un matériau isotrope. L'ensemble des valeurs obtenues varient de 1,67 à 4,25 (moyenne 2,2) et plus des deux tiers de celles-ci sont comprises entre 1,67 et 2,47 , leur valeur moyenne étant égale à 2,1.

L'argile se comporte donc comme un matériau anisotrope ou plus exactement comme un materiau orthotrope d'axe d'orthotropie vertical. Son comportement drainé, assimilé à un comportement élastique dans le domaine des faibles déformations, est défini par les paramètres suivants :

$E_{y}^{\prime}$, module d'Young vertical,

$E_{t}^{\prime}$, module d'Young dans le plan horizontal,

$v_{\text {in, }}^{\prime}$ coefficient de Poisson égal au rapport des déformations horizontale et verticale dues à une variation de contrainte normale verticale, $v_{h h}^{\prime}$, coefficient de Poisson égal au rapport des déformations suivant deux axes perpendiculaires $O x$, et $O x_{\text {, }}$ situés dans le plan horizontal dues à une variation de contrainte normale qui s'exerce suivant $O x_{2}$ $G_{v h}^{\prime}$, module de cisaillement dans le plan vertical.

Il s'ensuit que les paramètres $E^{\prime}$ et $v^{\prime}$ correspondent aux paramètres $E_{y}^{\prime}$ et $v_{v h}^{\prime}$. Toutes les éprouvettes ayant été découpées verticalement, il n'est, en principe, pas possible de déterminer les valeurs du rapport $n=\frac{E_{v}^{\prime}}{E_{h}^{\prime}}$ du rapport des modules et du coefficient de Poisson $v_{h i h}^{\prime}$. On peut toutefois obtenir un ordre de grandeur de celles-ci à partir du rapport $\frac{\varepsilon_{\mathrm{vc}}}{\varepsilon_{\mathrm{ac}}}$ si l'on admet la validité de la relation empirique:

$$
v_{h h t}^{\prime}=v_{v h}^{\prime}\left(2-\frac{1}{n}\right)
$$

établie par Atkinson (1975) pour l'argile de Londres.

$$
\text { L'expression de } \frac{\varepsilon_{\mathrm{vc}}}{\varepsilon_{\mathrm{ac}}} \text { en fonction des paramètres }
$$

d'élasticité orthotrope s'écrit en effet:

$$
\frac{\varepsilon_{\mathrm{vc}}}{\varepsilon_{\mathrm{ac}}}=\frac{2 \mathrm{n}\left(1-2 v_{\mathrm{hh}}^{\prime}\right)-4 v_{\mathrm{vh}}^{\prime}+1}{1-2 v_{\mathrm{vh}}^{\prime}}
$$

et, en la combinant avec la relation d'Atkinson, il vient :

$$
n=\frac{1}{2}\left(\frac{\varepsilon_{\mathrm{vc}}}{\varepsilon_{\mathrm{ac}}}-1\right)
$$

Si l'on retient pour $\frac{\varepsilon_{\mathrm{vc}}}{\varepsilon_{\mathrm{vc}}}$ et $v_{\mathrm{vh}}^{\prime}$ les valeurs moyennes

2,1 et 0,17 , on obtient $n=0,55$ et $v_{h+h}^{\prime}=0,03$, très proches de celles trouvées par Atkinson pour l'argile de

\begin{tabular}{|c|c|c|c|c|}
\hline Essais & $\begin{array}{l}\text { Modules de } \\
\text { déformation }\end{array}$ & $\mathrm{E}^{\prime}(\mathrm{MPa})$ & $\beta$ & $\begin{array}{c}E(M P a) \\
\text { pour } z^{*}=6 m\end{array}$ \\
\hline \multirow{4}{*}{$\begin{array}{l}\text { UU } \\
\text { et } \\
\text { CIU }\end{array}$} & $E_{\text {unas }}$ & 91,7 & 0,334 & 70 \\
\hline & $E_{\text {uns }}$ & 66,5 & 0,328 & 51 \\
\hline & $E_{u t}$ & 46,6 & 0,342 & 35,5 \\
\hline & $\mathrm{E}_{\text {uss }}$ & 72,4 & 0,306 & 56,5 \\
\hline \multirow{4}{*}{$\mathrm{CID}$} & $E_{0}^{\prime}$ & 72,8 & 0,306 & 56,5 \\
\hline & $E_{b s}^{\prime}$ & 46,1 & 0,298 & 36,2 \\
\hline & $E_{1}^{\prime}$ & 36,8 & 0,322 & 28,5 \\
\hline & $E_{s 0}^{\prime}$ & 32,5 & 0,28 & 26 \\
\hline
\end{tabular}
Londres $\left(n=0,5, v_{n h}^{\prime}=0\right)$.

TABtEAU VIt Paramètres de la relation empirique entre les modules de déformation en compression et la pression de consolidation. Valeurs de ces modules à une profondeur de $6 \mathrm{~m}$ dans l'argile.

Parameters of the empirical relationship between deformation moduli derived from compression tests and consolidation pressure. Values of these moduli at a depth of $6 \mathrm{~m}$ below the top of the clay layer. 


\section{Détermination à partir des essais au pressiomètre autoforeur}

Les valeurs du module de cisaillement $\mathrm{G}$ déterminées au pressiomètre autoforeur à différentes profondeurs ont permis d'obtenir un ordre de grandeur des valeurs correspondantes des modules $E^{\prime}$ et $E^{\prime}$ en tenant compte des paramètres d'élasticité orthotrope évalués précédernment. $G$ est en effet le module $G_{h+}$ dans le plan horizontal à la profondeur de l'essai et est lié au module horizontal $E_{h}^{\prime}$ par la relation :

$$
E_{h}^{\prime}=2\left(1+v_{h h}^{\prime}\right) G_{h h}=2(1+0,03) G=2,06 \mathrm{G} \text {. }
$$

Si on admet que le rapport des modules vertical et horizontal vaut $\mathrm{n}=0,55$ on peut aussi relier $\mathrm{G}$ à $E_{v}^{\prime}$ par la relation :

$$
E_{v}^{\prime}=2 \mathrm{n}\left(1+v_{h h}^{\prime}\right) G_{h h}=2 \times 0,55(1+0,03) G=1,13 \mathrm{G} .
$$

Les variations avec la profondeur du module vertical initial tangent $E_{\infty}^{\prime}$ correspondant au module de cisaillement initial tangent $G$ sont représentées sur la figure 18. En dépit d'une dispersion notable (liée à la dispersion de $G$ ), on constate une augmentation sensible de $E^{\prime}$ au-delà d'une profondeur de $5 \mathrm{~m}$ dans l'argile (les essais en place indiquent une tendance analogue pour la cohésion non drainée). Compte tenu de l'incertitude sur $n$ et $v_{h i}^{\prime}$ (qualité des éprouvettes, caractère empirique de la relation d'Atkinson), les valeurs de $E_{\text {vo }}^{\prime}$ ne peuvent être considérées que comme indicatives. Il semble cependant qu'elles marquent la limite inférieure du module vertical initial tangent. En effet, les informations disponibles concernant les paramètres d'élasticité orthotrope des argiles (Meftah et Magnan, 1988) indiquent que seules les argiles très fortement surconsolidées sont caractérisées par des valeurs de $n$ et de $v^{\prime}$ beaucoup plus faibles que celles retenues pour l'argile des Flandres (argile du Lias pour laquelle $n=0,21 ; v_{\text {th }}^{\prime}=$ 0,$19 ; v_{h h}^{\prime}=-0,38$ ). Dans ces conditions, $E_{v 0}^{\prime}$ ne peut qu'être supérieur aux valeurs de la figure 18, sa limite supérieure (sol isotrope, $n=1, v^{\prime}=0,17$ ) étant de l'ordre de deux fois celles-ci. En revanche, les valeurs de $E_{\text {ho }}^{\prime}$ peuvent être considérées comme représentatives du comportement de l'argile, l'erreur sur $v_{\text {hh }}^{\prime}$ étant faible.

La comparaison des modules $E_{\text {, }}^{\prime}$ et des modules $E^{\prime}$ obtenus à partir des essais triaxiaux $\mathrm{CID}$ a été faite à partir de la corrélation :

$$
E_{0}^{\prime}=72,8 \sigma_{c}^{0306}
$$

basée sur les résultats de ces essais (cf. § 7.1.2).

En dépit du caractère approximatif des valeurs de $E^{\prime}$ ainsi obtenues (qui ne peuvent évidemment rendre compte de l'hétérogénéité de l'argile en place), cette comparaison montre que celles-ci sont bien inférieures à $E^{\prime}$ ce qui confirme que les modules de déformation déterminés à partir d'essais classiques à l'appareil triaxial sont généralement sous-estimés (cf. § 7.1.1).

\section{8}

\section{Conclusion}

L'étude précédente, qui a mis en jeu la plupart des essais mécaniques réalisés couramment en laboratoire et en place, apporte de nombreuses informations sur les propriétés de l'argile des Flandres rencontrée à Dunkerque et à Calais sous un recouvrement de sable flandrien épais d'une trentaine de mètres.

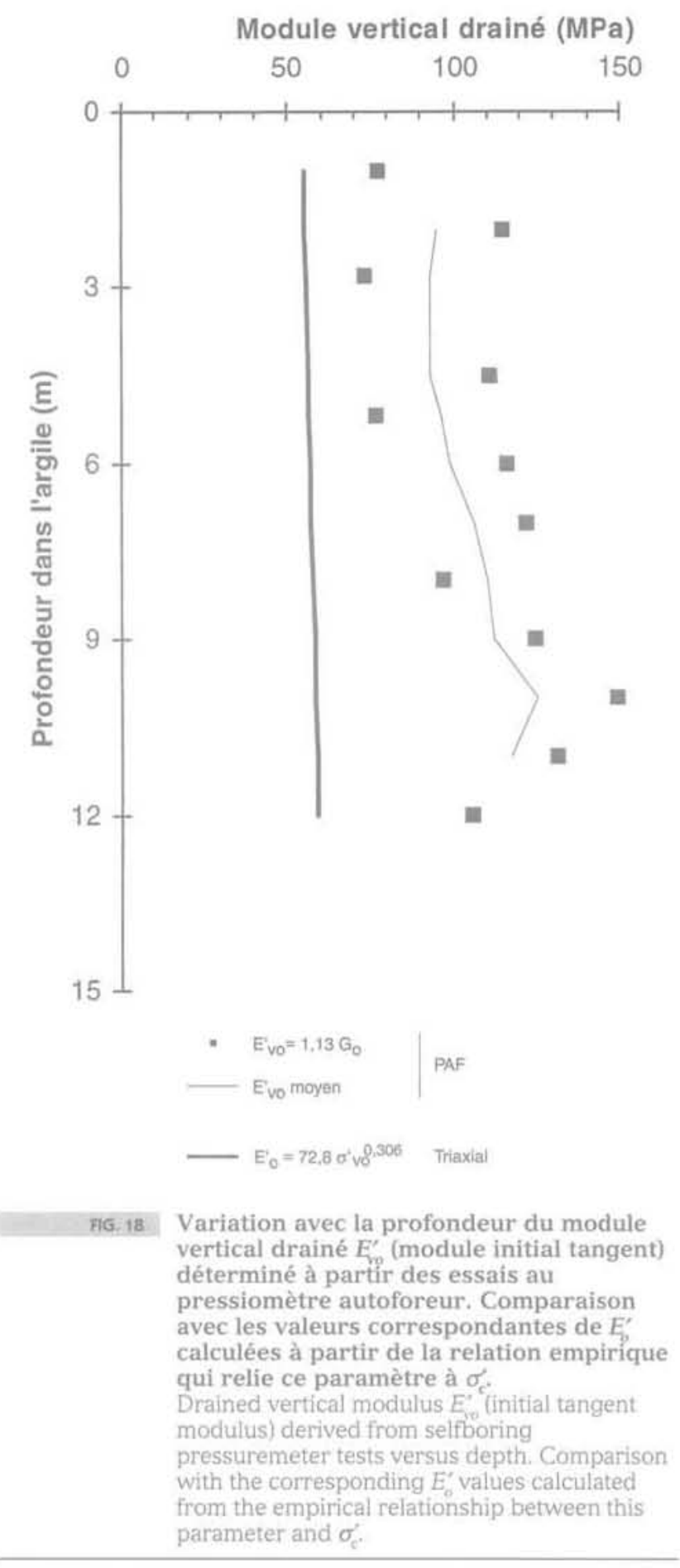

Cette étude a montré que les propriétés de l'argile étaient pratiquement identiques dans les deux sites étudiés et a permis de déterminer les principaux paramètres qui régissent son comportement ou d'obtenir des valeurs indicatives de ceux-ci. Les valeurs de ces paramètres sont récapitulées dans le tableau VIII.

Elle a, en outre, confirmé la validité de la relation empirique qui relie le rapport de surconsolidation et la cohésion non drainée $c_{u}$ d'une argile, relation dont on a déduit la loi qui régit les variations de $c_{u}$, lors du déchargement de l'argile. Des relations empiriques entre les modules de déformation de l'argile et sa pression de consolidation ont également été établies. 


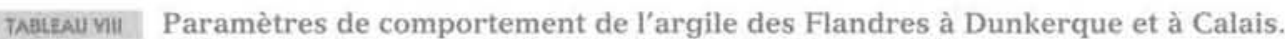

Soil parameters governing Flanders clay behaviour at Dunkirk and Calais.

\begin{tabular}{|c|c|c|}
\hline Paramètres & Valeurs & Commentaires \\
\hline Coefficient de pression du sol au repos & $0,9 \leq \mathrm{K}_{0} \leq 1,1$ & \\
\hline $\begin{array}{l}\text { Paramètres de résistance au cisaillement drainé } \\
\text { au pic } \\
\text { résiduel }\end{array}$ & $\begin{array}{c}c^{\prime} \leq 30 \mathrm{kPa}, \varphi^{\prime}=23^{\circ} \\
c_{r}^{\prime}=0, \varphi_{r}^{\prime}=14^{\circ}\end{array}$ & \\
\hline Cohésion non drainée & $200 \mathrm{kPa} \leq c_{\mathrm{u}} \leq 300 \mathrm{kPa}$ & \\
\hline $\begin{array}{l}\text { Paramètres de compressibilité } \\
\text { pression de préconsolidation } \\
\text { indice de compression } \\
\text { indice de gonflement }\end{array}$ & $\begin{array}{c}2,8 \mathrm{Mpa} \leq \sigma_{p} \leq 3,4 \mathrm{Mpa} \\
0,29 \leq C_{c} \leq 0,34 \\
0,10 \leq C_{n} \leq 0,12\end{array}$ & \\
\hline $\begin{array}{l}\text { Paramètres d'élasticité orthotrope } \\
\text { rapport d'anisotropie } \\
\text { coefficients de Poisson } \\
\text { module de cisaillement horizontal* } \\
\text { module de déformation horizontale drainé* } \\
\text { modules de déformation verticale non drainés* } \\
\text { modules de déformation verticale drainés* }\end{array}$ & 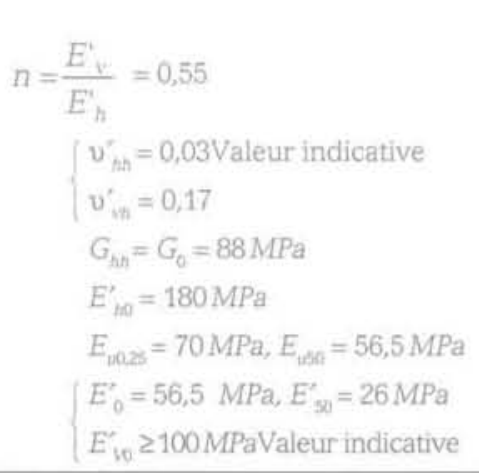 & Valeur indicative \\
\hline
\end{tabular}

*à une profondeur de 6 mètres dans l'argile.

On a aussi testé la fiabilité de la détermination de certains paramètres géotechniques à partir des essais en place les plus courants, dont l'interprétation est basée sur des corrélations empiriques. Dans le cas de l'argile étudiée, il apparait:

- que les essais au dilatomètre Marchetti exploités à partir des corrélations proposées par son concepteur conduisent à des valeurs assez réalistes de $\mathrm{c}_{\mathrm{u}}$ et de $K_{\mathrm{o}}$; - que les corrélations associées à l'essai au piézocône et à l'essai pressiométrique Ménard, qui donnent les valeurs de $c_{u}$ les plus voisines de celles déterminées au scissomètre, sont respectivement:

$$
c_{u}=\frac{p_{t}-\sigma_{v o}}{N_{k}} \text { avec } N_{k}=10 \text { à } 12
$$

et

$$
c_{u}=\frac{p_{1}-p_{0}}{5,5}
$$

la seconde conduisant d'ailleurs à des valeurs manifestement trop faibles. Ceci met en cause la validité des différentes corrélations habituellement proposées pour les argiles raides.

Enfin, il s'est avéré que la majorité des essais triaxiaux de type UU réalisés suivant la procédure classique sous-estiment considérablement la cohésion non drainée de l'argile. De tels résultats illustrent la nécessité de réaliser ces essais à partir de prélèvements de qualité, mais aussi d'adopter une procédure d'essai améliorée qui permettra notamment de contrôler la fiabilité des résultats obtenus.

\section{REMERCIEMENTS}

L'auteur tient à remercier tout particulièrement :

M. M. Dervaux, ingénieur au LRPC de Lille, pour les nombreuses informations que celuj-ci lui a transmises, ainsi que pour les intéressantes discussions quíl a eues avec lui;

MM. J.-P. Stempfelet et M. Daurade, techniciens supérieurs au LCPC pour leur importante contribution aux études expérimentales réalisées au LCPC;

M. L. Mespouthé, ATTPE au LCPC pour l'aide qu'ill lui a apportée dans la préparation de cet article. 
Amar S., Jézéquel J. - a Essais en place et en laboratoire sur sols cohérents. Comparaison des résultats 3 . Bulletin de Liafson des LPC, $\mathrm{n}^{\circ} 58$, mars-avril 1972 , p. $97-108$.

Amar S., Baguelin F., Jézéquel J., Le Méhauté A. $-\alpha$ In situ shear resistance of clays x. Proceedings of the Conference on in situ measurement of soil properties, Raleigh, vol. 1. June 1-4 1975, p. 22 45.

Atkinson J.H. - $\ll$ Anisotropic elastic deformations in laboratory tests on undisturbed London clay 13. Geotechnique, vol. $25, n^{\circ} 2,1975$, p. $357-374$

Baguelin F., Jézéquel J., Le Mée E., Le Méhauté A. - « Expansion de sondes cylindriques dans les sols cohérents $\gg$. Bulletin de Liaison des LPC. $n^{\circ}$ 61, septembre-octobre 1972, p. 189-202.

Baldi G.. Hight D.W., Thomas G.E. $-\alpha$ A reevaluation of conventional triaxial test methods 1. Advanced Triaxial Testing of Soll and Rock. ASTM STP 977, 1988, p. 219-263.

Berre T. - ir Triaxial testing at the Norvegian Geotechnical Institute 1.. Oslo. Publication $\mathrm{n}^{\circ} 134,1981$

Bishop A.W., Henkel D.J. - The measurement of soil properties in the triaxial test. 2nd ed, Edward Arnold, London, 228 p., 1962.

Boulinet J.-C. Waschkowski E. - « Étude expérimentale et analyse des performances đu pénétro-dilatomètre Marchetti ». Compte rendu de travail. Laboratoire régional des ponts et chaussées de Blois, 1994.

Cassan M. - Les essais in situ en Mécanique des sols. Tome 1, Réalisation et interprê.

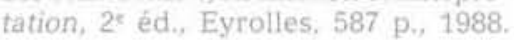

Combarieu O. - \& L'essai pressiométrique et la résistance au cisaillement des sol D. Bulletin de Liaison des LPC, $n^{\circ} 196$, mars-avril 1995, p. 43-51.

Hieng O.I - $\alpha$ Loi de comportement d'une argile raide (détermination des paramètres géotechniques de l'argile des Flandres) $)$. Thèse de doctorat de l'université de Paris 6, 259 p.. 1991.

Jardine R.J. Symes M.J. Burland J.B. «T The measurement of the soll stiffness in the triaxial apparatus ग. Geotechnique, vol. $34, n^{\circ} 3.1984$, p. 323-340.

IFP - "Port de Calais. Essais géotechniques et sismiques \%. Rapport de l'Ins. titut Français du Pétrole, 1992.

Josseaume H. Hieng O.I. Stempfelet J.P. a Détermination des paramètres de compressibilité d'une argile raide à partir d'essais cedométriques â haute pres. sion $\gg$. Bulletin de Liaison des LPC. $n^{\circ} 172$, mars-avril 1991. p. 109-120.

Lacasse S. Berre T. - a Triaxial Testing Methods for Soils D. Advanced Triaxial Testing of Soil and Rock. ASTM. STP 977, 1988, p. 264-289.

Ladd C.C. - " Stress-strain behaviour of anisotropically consoliclated clays during undrained shear 13 . Proceedings of the 6 th International Conference on Soil Mechanics and Foundation Engineering, Montréal, vol. 5, 1965, p. 282-286.

Ladd C. C. Foot R. Ishihara K. Schlosser F. Poulos H.G. - "Stress-deformation and strength characteristics $\gg$. Proceedings of the 9th International Conference on Soil Mechanics and Foundation Engineering, Tokyo, vol. 2. 1977, p. 421-494.

Marchetti S. - $\alpha$ In situ tests by flat dilatometer w. ASCE, Journal of the Geotechnical Engineering Division, vol. 106 $n^{\circ}$ GT 3, March 1980, p. 299-321.
Mayne P.W. - " Stress anisotropy effects on clay strength \%. ASCE, Journal of the Geotechnical Engineering, vol. 111. $n^{\circ}$ G 3,1985 , p. 356-366.

Mayne P.W., Kulhawy F.H. - « KoCA relationships in solls ม. ASCE, Journal of the Geotechnical Engineering Division, vol. 108, n GT 6, June 1982, p. 851-872.

Meftah K., Magnan J.-P. - is Anisotropie de la résistance, de la déformabilité et de la perméabilité des sols. Etat des connais. sances 3). Rapport des laboratoires. Série : Géotechnique, Mécanique des sols, Sciences de la Terre, GT 31. Laboratoire Central des Ponts et Chaussées. 101 p., 1988

Mitachi T. Kitago S. - $\alpha$ Change in undrained shear strength characteristics of saturated remoulded clays due to swel-

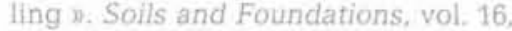
$n^{*} 1$, March 1976, p. $45-58$

Neveu C., Piet E., Delattre L. - a Calais quai en eau profonde ». Compte rendu du Congrès de l'AIPCN, Séville. Section II, Sujet 3, 1994, p. 59-64.

Port Autonome de Dunkerque - « Le terminal à pondéreux ouest ». Chantiers, $n^{\circ} 146,1981$, p. $3-8$

Skempton A.W. - $*$ Horizontal stresses in an overconsolidated Eocene clay o. Proceedings of the 5 th International Conference on Soil Mechanics and Foundation Engineering, Paris, vol. 1, 1961. p. $351-357$.

Webb D.L. - $-\alpha$ Residual strength in conventional triaxial tests 1 . Procee. dings of the 7 th International Conference on Soil Mechanics and Foundation Engineering, Mexico, vol. 1, 1969, p. $433-441$ 